\title{
Online Energy Management Systems for Microgrids: Experimental Validation and Assessment Framework
}

\author{
Adriana C. Luna, Student, IEEE, Lexuan Meng, Member, IEEE, Nelson L. Diaz, Moisès Graells, Juan C. \\ Vasquez, Senior, IEEE, and Josep M. Guerrero, Fellow, IEEE
}

\begin{abstract}
Microgrids are energy systems that can work independently from the main grid in a stable and self-sustainable way. They rely on energy management systems to schedule optimally the distributed energy resources. Conventionally, the main research in this field is focused on scheduling problems applicable for specific case studies rather than in generic architectures that can deal with the uncertainties of the renewable energy sources. This paper contributes a design and experimental validation of an adaptable energy management system implemented in an online scheme, as well as an evaluation framework for quantitatively assess the enhancement attained by different online energy management strategies. The proposed architecture allows the interaction of measurement, forecasting and optimization modules, in which a generic generation-side mathematical problem is modeled, aiming to minimize operating costs and load disconnections. The whole energy management system has been tested experimentally in a test bench under both grid-connected and islanded mode. Also, its performance has been proved considering severe mismatches in forecast generation and load. Several experimental results have demonstrated the effectiveness of the proposed EMS, assessed by the corresponding average gap with respect to a selected benchmark strategy and ideal boundaries of the best and worst known solutions.
\end{abstract}

Index Terms-Energy management, Power generation dispatch, Reconfigurable architectures, Technology assessment

\begin{tabular}{|c|c|c|}
\hline \multicolumn{2}{|c|}{ NOMENCLATURE OF THE OPTIMIZATION PROBLEM } & $X$ \\
\hline$k$ & Index for discrete time. & \\
\hline$i$ & Index for generation units. & $J(X, Z)$ \\
\hline$j$ & Index for storage units. & $J 1(X, Z)$ \\
\hline$K$ & Number of time slots. & \\
\hline$n_{i}$ & Number of energy sources. & $J 2(X, Z)$ \\
\hline$n_{j}$ & Number of energy storage systems. & \\
\hline$\Delta t$ & Duration of time slot $[\mathrm{s}]$ & \\
\hline$T$ & Time horizon of scheduling [s]. & $J 3(X, Z)$ \\
\hline$C(i, k)$ & Unitary cost of energy sources [€/kWh]. & $P_{g}(i, k)$ \\
\hline$\xi_{g}(i, k)$ & $\begin{array}{l}\text { Elementary penalization cost for not using } \\
\text { energy sources at slot } k[€ / \mathrm{kWh}] \text {. }\end{array}$ & $P_{E S S}(j, k)$ \\
\hline$\xi_{\text {load }}(k)$ & $\begin{array}{l}\text { Elementary penalization cost for not sup- } \\
\text { plying the load at slot } k[€ / \mathrm{kWh}]\end{array}$ & $S o C(j, k)$ \\
\hline & $\begin{array}{l}\text { g, J. C. Vasquez and J.M. Guerrero are with the } \\
\text { echnology, Aalborg University, } 9220 \text { Aalborg, Den- }\end{array}$ & $\begin{array}{l}z_{\text {load }}(k) \\
\hat{d}(i)\end{array}$ \\
\hline
\end{tabular}
(reprint author),juq@et.aau.dk, joz@et.aau.dk (see http://www.microgrids.et.aau.dk).

N. L Diaz is with the Engineering Faculty, Universidad Distrital Francisco Jose de Caldas, Bogota 110231, Colombia, email: nldiaza@udistrital.edu.co

M. Graells is with Chemical Engineering Department, Universitat Politècnica de Catalunya (UPC), E-08019 Barcelona, Spain, email: moises.graells@upc.edu

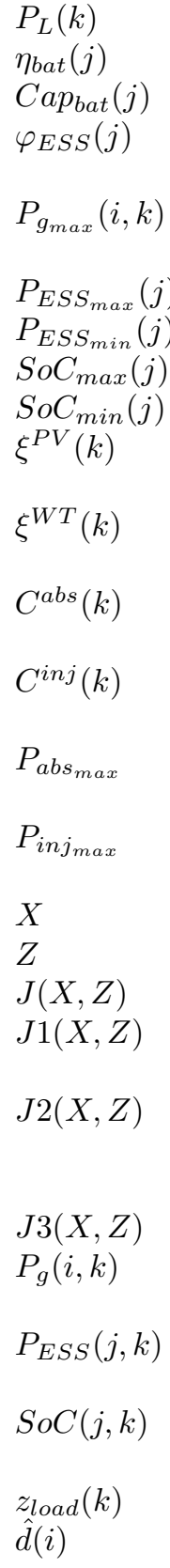

Power of the load at each $k[\mathrm{~kW}]$. Charge/discharge efficiency of $j$-th ESS. Capacity of the $j$-th storage unit [kWh]. Coefficient associated to the $j$-th ESS $[\% / \mathrm{kWh}]$. Maximum generation power of the $i$-th energy source at the $k$-th slot [kW]. Maximum power of the $j$-th ESS [kW]. Minimum power of the $j$-th ESS [kW]. Maximum SoC of the $j$-th ESS [\%]. Minimum SoC of the $j$-th ESS [\%]. Elementary penalization cost for not using PV energy at slot $k$ [€/kWh].

Elementary penalization cost for not using WT energy at slot $k$ [€/kWh].

Elementary cost for absorbing energy from the main grid at slot $k[€ / \mathrm{kWh}]$.

Elementary cost for injecting energy to the main grid at slot $k$ [€/kWh].

Upper boundary for absorbing power from the main grid $[\mathrm{kW}]$.

Upper boundary for injecting power from the main grid [kW].

Real decision variables.

Binary decision variables.

Objective function.

Operating cost for using the energy sources (main grid in the selected case study).

Penalization for not using energy sources with no operating cost (RESs in the case study).

Penalization for not supplying the load. Average power of the $i$-th energy source at the $k$-th slot $[\mathrm{kW}]$.

Average power of the $j$-th storage unit at the $k$-th slot $[\mathrm{kW}]$.

State of Charge of the $j$-th storage unit at the $k$-th slot [\%].

Activation of the load at the $k$-th slot [kW]. Power flow direction of the $i$-th generation units, i.e. $\hat{d}(i)=1$ or $\hat{d}(i)=-1$ if injected or absorbed power to/from the $\mathrm{MG}(\hat{d}(i)=$ -1 only for power injected to the grid in case study). 


\section{INTRODUCTION}

A S commonly recognized, a microgrid (MG) is an energy system composed of loads and Distributed Energy Resources (DER), such as Distributed Generators (DG) and Energy Storage Systems (ESS), that can operate either in islanded or grid-connected configuration [1]-[3]. The current technology based on power electronic interfaces allows an easy integration of Renewable Energy Sources (RES) in MGs, which combined with ESSs can provide economic benefits, while ensuring an efficient use of RESs and reliable load supply [4], [5]. However, their implementation and coordination within an MG imply a number of challenges on control and management due to the variability and uncertainty of RES [6], [7]. In this context, the optimal operation scheduling of MGs becomes critical to achieving uninterrupted supply and economic objectives [8]. The scheduling function in MGs is performed by an energy management level, which should operate in a lower bandwidth than control and power management levels, in order to have a low impact on the stability and robustness performance of the MG [2]. The control and power management strategies affect the instantaneous operational conditions related to current, voltage quality and frequency regulations, as well as active/reactive power dispatching power sources oriented to the feasibility of the system and, optionally, provide ancillary services [3], [9]-[11]. Particularly, power management level should be in charge of dealing with issues that can affect the performance of the system, like line limits, losses, converter droops, as well as autonomous operation strategies, as presented in [12]-[16].

In turn, the energy management ensures the availability of the resources during longer time intervals, matching the total power production to the demand in an optimal way [17]. Accordingly, the energy management system of the MG (MGEMS) establishes the reference commands for DER/loads controllers to make the whole system operate optimally, not only considering the current MG behavior, but also economical and technical constraints, environmental conditions, and external requirements [18], [19]. To do that, the MG-EMS usually performs a short-term scheduling process for operation, which uses input parameters and profiles involving the prediction of available resources subjected to uncertainties [20].

Extensive researches have been recently reported related to the management of MGs, mainly focused on mathematical formulations and usually tested under deterministic and offline scenarios, as reviewed in [21]. For instance, in [22], a linear mathematical model is suggested to balance the generation and load of a hybrid microgrid by minimizing the total operating cost of the system in a 24-hour period. In [23], a control combines fuzzy control with gain scheduling techniques to accomplish both power sharing and energy management. In [24], an economic dispatch problem for total operation cost minimization in DC microgrids is formulated and solved with a heuristic method. However, these approaches do not deepen in the design of the MG-EMS architecture so that it can be adaptable and easily integrated into the power system. In this regard, a smart energy management system is defined in [25] with an architecture that uses different functional mod- ules, which are connected sequentially, but this can produce bottlenecks in the data flow.Additionally, the previous works do not deal with the uncertainty of the RES generation and consumption. In this sense, online strategies have been implemented such as in [26]-[31], where MG-EMSs are designed and implemented by considering the current status of the MG, but without foreseeing the future availability of generation or consumption. In [32], an online optimal energy/power control method is presented for the operation of energy storage in gridconnected electricity microgrids considering predicted future electricity usage and renewable energy generation but they do not consider safety ranges and proper charging stage for the operation of ESS based on batteries, which ensures longer ESS lifetime [33]. In [34], a rolling horizon strategy is defined for a specify case study while in [35], an online MG-EMS focused on the optimization process presented. In [36], [37], centralized MG-EMSs for isolated microgrids are designed by decomposing the unit commitment and optimal power flow in order to avoid a mixed-integer non-linear formulation. However, most of these proposals are not tested experimentally and also the optimization model is not defined to be used in both grid-connected and islanded operation modes. In [38], an EMS for both modes is proposed in a two-layer structure using a linear model but the system is just validated in simulation.

Moreover, such investigations assess the performance of their proposals by comparing to those obtained in one or several limited benchmarks. Yet, they lack an assessment framework that quantitatively evaluates the absolute improvement against the worst case and the gap between the performance attained and the best possible case. Thus, no hint is provided in regard to the extent to which the improvement is significant and the extent to which further improvement is still achievable. For instance, the MG-EMS presented in [35] is compared with two benchmarks, one greedy strategy and one offline approach, but the validation strongly depends on the selected cases.

In this paper, an adaptable online MG-EMS is designed in order to deal with the variability introduced by renewable generators. The proposal is experimentally tested in an $\mathrm{MG}$ under grid-connected and islanded modes and an evaluation framework is defined for quantitatively assess the enhancement attained by different online energy management strategies. The MG-EMS design is based on a flexible architecture that includes four modules, which perform the functions of scheduling, processing, forecasting, and acquisition data, respectively. The scheduling process relies on a generalized mathematical formulation that addresses the economic management of an MG, either in grid-connected or islanded operation. In turn, the processing module dynamically adjusts the outcomes of the forecasting module by using the available measurements in order to make the system less vulnerable to prediction errors.

The proposed MG-EMS is experimentally tested in a laboratory scale microgrid by defining an MG case study that includes RESs and a battery as an ESS. Its operation is tested in both operation modes of the MG, considering normal uncertainties in the weather prediction, but also under big mismatches of the prediction in islanded mode, such as an unexpected no production of WT generation and non-scheduled load disconnection. 


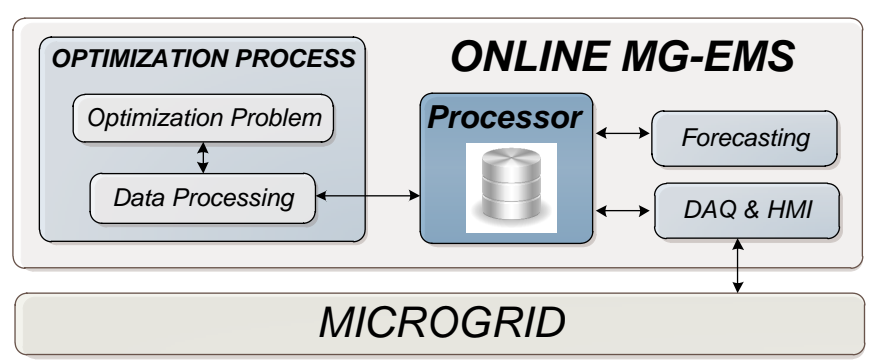

Fig. 1. General Scheme of the Proposed Online MG-EMS

The validation of the proposal is assessed by the corresponding average gap with respect to a selected benchmark strategy and ideal boundaries of the best and worst solutions. Hence, giving a set of scenarios, the operational cost is obtained by using the different MG-EMS approaches, namely, the benchmark case, the proposed MG-EMS, and the ideal references. Subsequently, the quantitative analysis of the results provides an overview of the improvement achieved by implementing the proposed MG-EMS over the selected case, but also the margin of improvement that still can be achieved.

\section{Proposed Energy Management System}

\section{A. Online Architecture}

The modular architecture, shown in Fig. 1, is proposed in order to define the references of the energy resources in a microgrid considering the variability of the energy resources.

This MG-EMS is composed of four modules. To start with, the interface between the MG and the MG-EMS is established by means of the Data Acquisition and Human Machine Interface module, $D A Q \& H M I$. Through this module, the user can monitor the system and modify input parameters. This unit is operating continuously, collecting the data for visualization and sending the reference data for the controller of the MG.

The Processor is the central unit that coordinates the interaction of the other modules to perform the scheduling process. It contains a data storage in which the output data of the modules are stored, as well as relevant parameters. For instance, for the operation of the Forecasting and the Optimization Process, global parameters should be defined for continuous and discrete time frameworks, as presented in Fig. 2. Some of these parameters are the time horizon, $T$, the duration of the time slots, $\Delta t$, how frequently the optimization will be run, $t_{r e p}$ and the number of time slots, $K$.

The forecasting module can work online, as well, in order to update the data related to energy generation and consumption. The forecast data is saved in the data storage to be available to next iterations of the optimization process. These data represent an estimation of the future conditions and always will have some errors for the defined time slots, $\delta_{1}, \delta_{2}, \ldots, \delta_{k}$, that increase depending on how close they are to the time when the forecasting is performed (Fig. 2).

The optimization process is, in fact, the module of the MGEMS that sets the references for the devices of the MG by taking into account the objective function defined by the user. This module works only under the request of the processor.

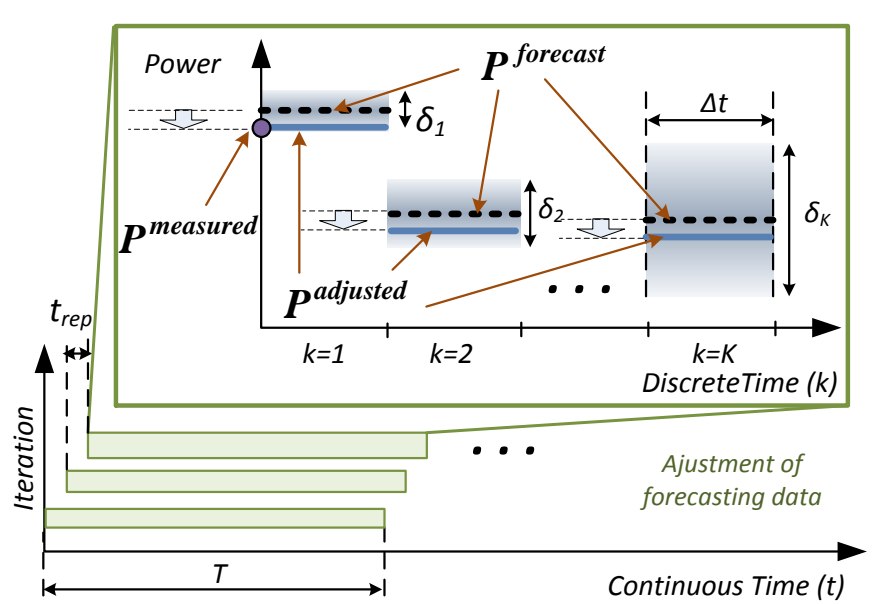

Fig. 2. Adjustment of Forecasting Data in each Iteration

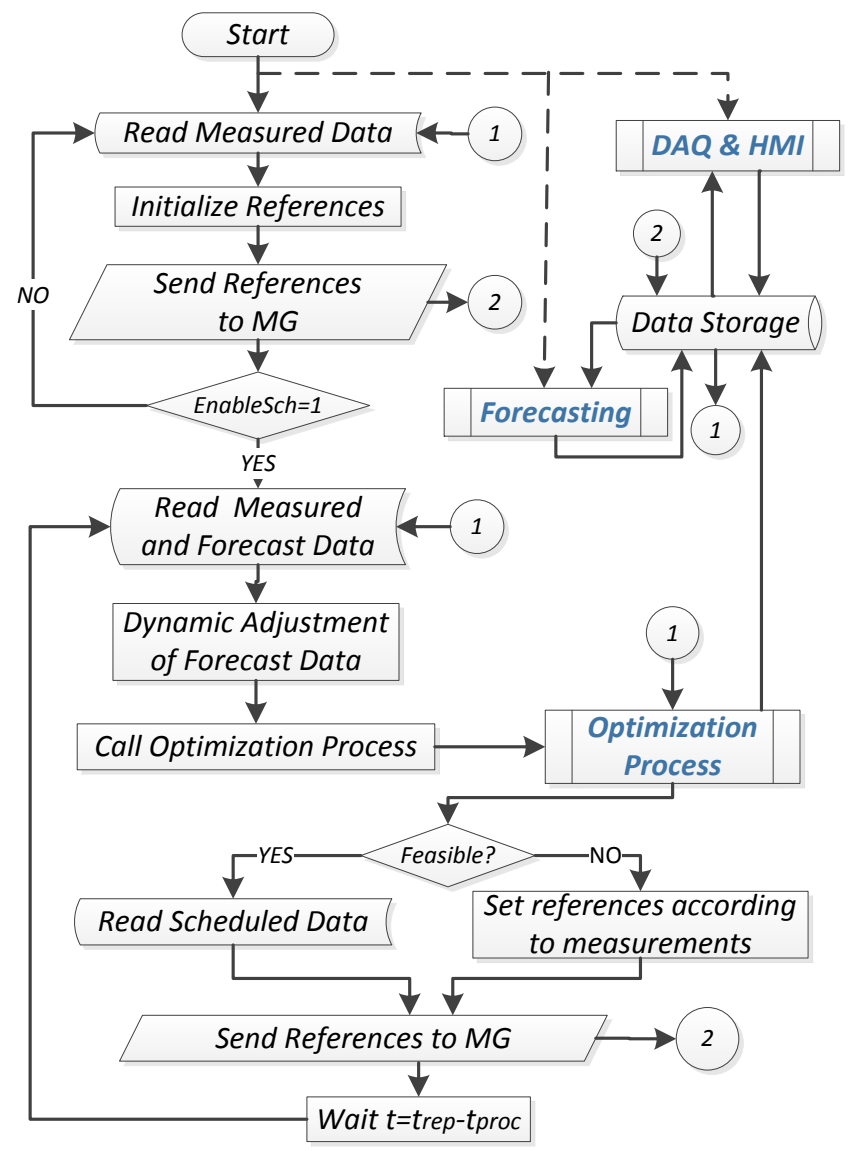

Fig. 3. Flow Diagram of the Processor

\section{B. Interaction of the Modules}

In the proposed MG-EMS, the Processor integrates the other modules by performing the actions summarized in Fig. 3. When the process starts, the modules $D A Q \& H M I$ and Forecasting can be initialized and synchronized with other modules. These two modules are saving the measured and the predicted data in the Data Storage as frequently as they can.

Meanwhile, the Processor reads the measured data that are available in the data storage. This dataset is used to 
define the reference for the local controllers of the MG. In general, the power references are initialized to $0 \mathrm{~kW}$ for the generation units that imply any operating costs. The power of the generation units with no operating costs are not limited, thus the references for the local controllers are equal to the measured data. The power of the ESSs is set to $0 \mathrm{~kW}$ as well, whereas all the loads are connected.

The references are stored in the Data Storage and read by $D A Q \& H M I$ to send them to the MG. In this way, the MG is provided with initial non-optimal references to operate by relying on its local controllers. The MG should have implemented contingent actions to manage fast critical events that can damage the MG components. These steps are repeated until the scheduling process is enabled (EnableSch $=1$ ).

To start with the scheduling process, the Processor reads the forecast data of the energy generation and consumption from the data storage. Additionally, it requires some data of the current situation of the MG, such as the currently available energy capacity of the ESSs, typically defined by their State of Charge (SoC), but also the average power values, which should be calculated before running the scheduling process. These data are taken and processed by the $D A Q \& H M I$.

The forecast data is adjusted dynamically by using the difference between the last measured average data and the value of the first time slot of the forecasting. This value is subtracted in every forecast value for the current time horizon, as can be seen in Fig. 2. The adjusted data will be used in the optimization iteration as input data.

After that, the Processor calls the Optimization Process to solve the optimization problem by collecting the previous data and some parameters that can be read directly from the data storage. The input and output data of the Optimization Process should be formatted, usually as data structures, so that they can be readable by the optimization problem. The results are saved in the data storage and sent to the MG by means of $D A Q \&$ $H M I$. In the case of infeasible solutions, the system should set provisional references according to DG power measurements and the SoC of the ESSs.

The scheduling process is repeated every $t_{r e p}$, which should be selected to make the MG-EMS work in a lower bandwidth than the local controllers. The process can be stopped or finalized at any moment of the sequence while the $D A Q \&$ HMI holds the last references to the MG.

\section{Optimization Problem}

The core of the MG-EMS is the optimization problem which optimizes the selected objective function by setting decision variables. In this case, a generation-side formulation is defined as a deterministic problem to minimize the operation cost and load disconnections in an MG composed by $n_{i}$ energy resources, $n_{j}$ ESSs and a load.

1) Statements: The proposed model is defined by assuming $k$ as the time in a discrete representation framework. As the time horizon is $T$ and the interval of each time slot is $\Delta t$, there are $K=T / \Delta t$ time slots.

Additionally, for each time slot, the energy of the devices is defined as, $P(k) \Delta t$ where the power is the average value in the interval $\Delta t$. The indexes related to the devices are $i$ for the generation units and $j$ for the ESSs.

Particularly, the problem includes the real and binary variables in term of the time $k, X$ and $Z$, defined as,

$$
\begin{aligned}
X & =\left[\begin{array}{lll}
P_{g}(i, k) & P_{E S S}(j, k) & S o C(j, k)
\end{array}\right], \\
Z & =\left[\begin{array}{l}
z_{\text {load }}(k)
\end{array}\right]
\end{aligned}
$$

where $P_{g}(i, k)$ is a matrix that aggregates the power of the $n_{i}$ energy resources at each $k, \operatorname{PESS}(j, k)$ is the matrix of the powers of the $n_{j}$ ESSs at each $k, S o C(j, k)$ is the matrix of the SoC for the $n_{j}$ ESSs at each $k$, and $z_{\text {load }}(k)$ is the binary variable for connection/disconnection of the load at each $k$. If a generation unit allows a bidirectional exchange of power, two superposed variables can be considered in $P_{g}(i, k)$, in order to model the injection and absorption of power, separately. In this way, differentiated costs can be assigned at each direction. Since the problem is modeled as a linear formulation, it is expected that the optimization process does not schedule power injection and absorption at the same time slot. In any case, this is a mathematical model which does not imply that physically the generation unit can inject and absorb power at the same time slot. The physical variable is the power of the unit which can be computed as the superposition of the auxiliary variables.

2) Mathematical formulation: As mentioned previously, the objective function $J(X, Z)$ is oriented to economic aspects and minimization of load disconnections. In this way, the proposed function can be written as,

$$
\begin{array}{rl}
\min _{X, Z} & J(X, Z)=J_{1}(X, Z)+J_{2}(X, Z)+J_{3}(X, Z)= \\
& \sum_{k=1}^{K} \sum_{i=1}^{n_{i}} C(i, k) *\left\{P_{g}(i, k) \Delta t\right\}+ \\
& \sum_{k=1}^{K} \sum_{i=1}^{n_{i}} \xi_{g}(i, k) *\left\{\left[P_{g_{\max }}(i, k)-P_{g}(i, k)\right] \Delta t\right\}+ \\
& \sum_{k=1}^{K} \xi_{\text {load }}(k) *\left\{\left(1-z_{\text {load }}(k)\right) P_{L}(k) \Delta t\right\}
\end{array}
$$

where $J_{1}(X, Z)$ is the operating cost or revenue for using the generation units, $J_{2}(X, Z)$ is a penalization for limiting the energy with no operating costs and $J_{3}(X, Z)$ is a penalization for not supplying the load. In turn, $C(i, k)$ is the unitary cost for using the energy from each $i-t h$ generation unit. This parameter can be positive or negative, admitting to define either the unit operating cost or the unit profit, respectively. $\xi_{g}(i, k)$ and $\xi_{\text {load }}(k)$ are penalization coefficients to assign priority for its associated term. Although their selection depends on the case, it is expected that $\xi_{\text {load }}(k)$ will have the highest value to prioritize power supply, as in [7]. $P g_{\max }(i, k)$ is the upper boundary of the energy sources power that can be variable in terms of $k$. Meanwhile, $P_{L}(k)$ is the load power profile.

Together with the objective function, some constraints should be defined to allow the problem to get feasible so- 
lutions. To start with, the energy balance in the microgrid has to be fulfilled. This constraint can be written as,

$$
\begin{aligned}
& \sum_{i=1}^{n_{i}} P_{g}(i, k) \hat{d}(i) \Delta t+\sum_{j=1}^{n_{j}} P_{E S S}(j, k) \Delta t= \\
& P_{L}(k) z_{\text {load }}(k) \Delta t, \quad \forall k
\end{aligned}
$$

where $\hat{d}(i)$ is a vector that specifies the power flow direction of each generation unit. In this way, some energy sources those allows bidirectional operation can be modeled.

Regarding the ESSs, their SoCs are defined as,

$$
\begin{aligned}
& S o C(j, k)=\operatorname{SoC}(j, k-1)- \\
& \quad \varphi_{E S S}(j)\left[P_{E S S}(j, k) \Delta t\right], \quad \forall k, j
\end{aligned}
$$

where $\varphi_{E S S}(j)$ is a coefficient associated with the physical features of each ESS. In the case of batteries, it is related to the efficiency of charging/discharging, $\eta(j)$, and the capacity in $\mathrm{kWh}, \operatorname{Cap}(j)$, which are provided by the manufacturers. This coefficient can be defined as,

$$
\varphi_{E S S}(j)=\frac{\eta_{b a t}(j)}{\operatorname{Cap}_{b a t}(j)} * 100 \%
$$

And finally, each variable must be bounded so that the problem can provide feasible solutions. Therefore,

$$
\begin{array}{cl}
0 \leq P_{g}(i, k) \leq P_{g_{\max }}(i, k), & \forall k, i \\
P_{E S S_{\min }}(j) \leq P_{E S S}(j, k) \leq P_{E S S_{\max }}(j), & \forall k, j \\
\operatorname{SoC}_{\min }(j) \leq S o C(j, k) \leq \operatorname{SoC}_{\max }(j), & \forall k, j \\
z_{\text {load }}(k) \in\{0,1\}, & \forall k
\end{array}
$$

where $P_{E S S_{\min }}(j)$ and $P_{E S S_{\max }}(j)$ are the boundaries of the ESS power. These parameters are provided by the manufacturers and determine the maximum power for charging and discharging the ESS. $S o C_{\min }(j)$ and $S o C_{\max }(j)$ are the boundaries for the $S o C$ of the battery.

In this way, the optimization problem described by the equations $3,4,5,7,8,9$ and 10 can be used in many microgrid structures to minimize operation costs.

\section{CASE STUdy}

The microgrid presented in Fig. 4 is selected as case study to validate the proposed MG-EMS. This MG is composed of two kinds of RES generation units and one battery that supply a common load bus. Voltage-source converters are used for interfacing the DG and the ESS with the microgrid [18]. The whole system will be tested in grid-connected mode and in islanded mode, independently.

\section{A. Operation of the Microgrid}

As shown in Fig. 4, the local controller of the ESS has different operational modes depending on whether the MG is connected to the main grid or is working in islanded mode.

When the system works in grid-connected mode, the main grid imposes the voltage and frequency conditions. Therefore, all the DG and the ESS work as grid-following units perfectly synchronized with the AC voltage at the connection point and controlled as current sources with an inner current control loop

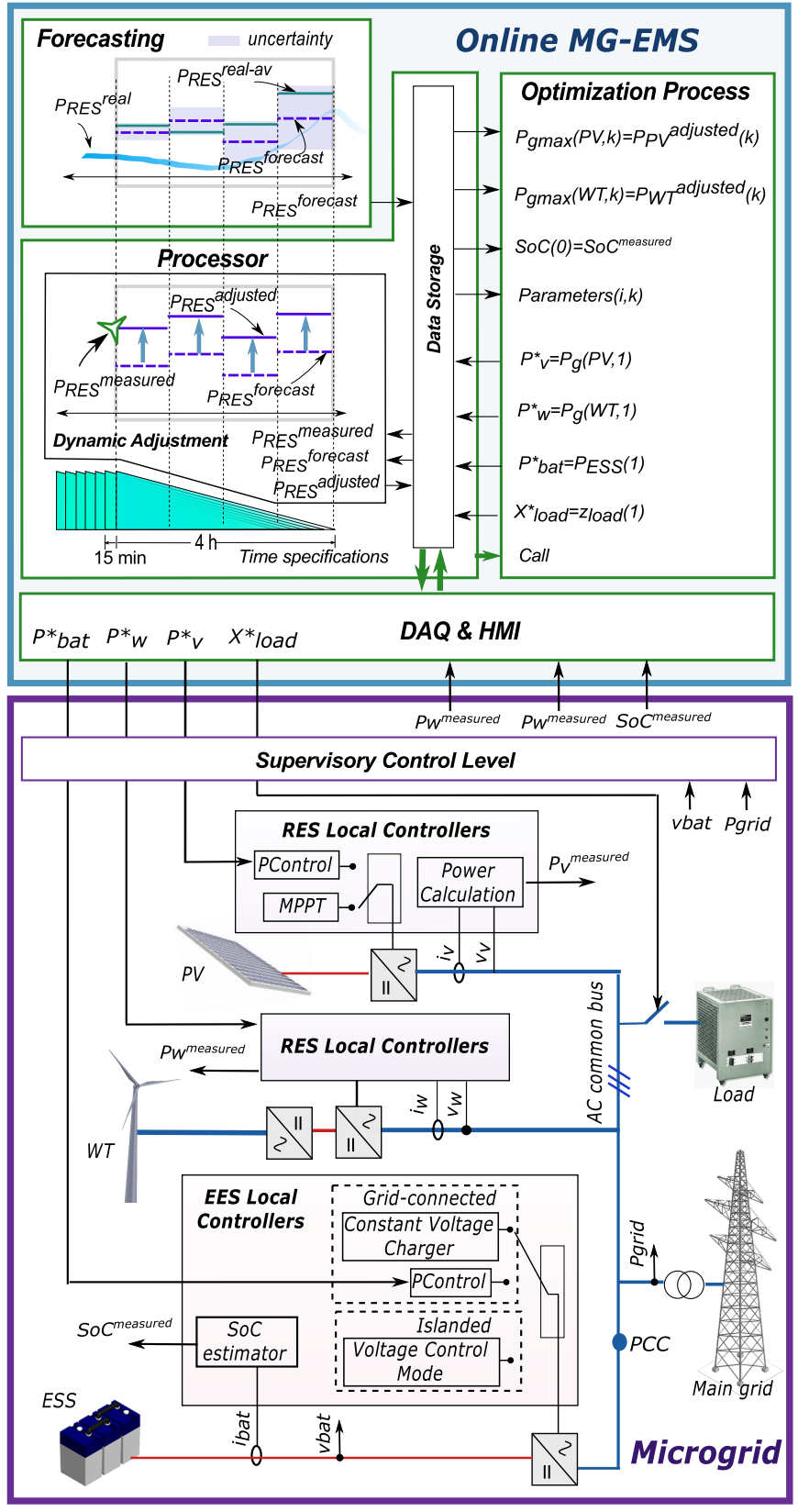

Fig. 4. Scheme of the selected case study with the online MG-EMS

in $d q$ reference frame [39]. The references of active power $\left(P^{*}\right)$ to be delivered or absorbed by the DG and the ESS can either be dispatched by the MG-EMS (PControl mode in Fig. 4), or defined locally by means of controllers that not interact with the MG-EMS. In the case of RESs, the noninteractive operation is activated when the reference provided by the MG-EMS is greater than the maximum available energy, then, the power reference is taken from a Maximum Power Point Tracking (MPPT) algorithm. In the case of the ESS, the non-interactive operation is activated when the battery in the ESS reaches its maximum limits of charge, which is defined by the manufacturers as a threshold voltage value $V_{t h}$. Under this condition, the reference of the inner current controller is defined by an outer battery voltage controller, which keeps the battery voltage in a constant level [19]. This charging 
stage is known as Constant-Voltage charger mode and is highly recommended for ensuring an appropriate charge of the battery [33]. During this stage, the battery current will taper slowly to keep the battery voltage at a constant value, while the battery is being saturated for ensuring a full charge. Thus, the ESS cannot follow the dispatched power reference. The ESS will change its operation mode when energy support is required.

In islanded mode, the ESS assumes the responsibility of compensating the unbalances between consumption and demand based on its inherent capability of absorbing or injecting energy, then, the ESS works as the grid-forming unit [18]. In this case, the inverter operates in voltage control mode and the inner current controller is complemented by an outer voltage control loop which regulates the voltage in the islanded power grid to match the reference value [39]. Because of that, during islanded operation, the power managed by the ESS cannot be directly dispatched by the MG-EMS, but the power and dis/charging rate can be limited indirectly by dispatching the power of the DG [40]. In contrast, RESs operate as gridfollowing units regardless the operational mode of the MG.

The inner current control loop is the same under all the different operational modes, just the outer controllers and reference generation units change between different operation modes. Seamless transitions between operational modes can be achieved by setting the initial conditions of the inactive controllers to the current value of the reference current. Interested readers may refer to [18], [19], [39] for detailed information about the different control loops.

Moreover, contingency actions are defined in the supervisory control level in order to ensure safety voltage levels of the ESS in the case of unexpected operation of the system. Therefore, if the battery voltage $v_{b a t}$ is lower than the End Of Discharge $(E O D=680 \mathrm{~V}$, for the selected VRLA battery and established by the manufacturer), the load is disconnected. Likewise, if $v_{b a t} \geq V_{t h}$ (in this case, $V_{t h}=756 \mathrm{~V}$ ), the generation from RES is progressively set to $0 \mathrm{~kW}$ for avoiding overcharge the battery. Normal operation is restored when the $S o C$ reaches the midpoint of its nominal operation range ([50\%,100\%]), i.e. $S o C=75 \%$, so that the ESS has an acceptable margin of maneuver to control the system. Also, in grid-connected mode, the supervisory level can limit the power injected to the main grid when the maximum limits are reached by modifying the curtailment of the RES.

\section{B. $M G-E M S$}

The MG-EMS explained in section II is implemented for the case study, as presented in Fig. 4. As mentioned before, the commands for the MG controllers are computed every $t_{r e p}=15$ min by the optimization process of the online MGEMS. The results are stored in the Data Storage and sent to the MG by the DAQ \& HMI module. The time horizon for the forecasting data and the optimization problem has been set to $T=4 \mathrm{~h}$ with four time-slots $(\Delta t=1 \mathrm{~h})$. This time horizon has been selected since most of the short-time forecasting are accurate up to this prediction time, even with persistence method [41]. The forecasting process has been emulated by adding random errors to the deterministic data, $P_{R E S}^{r e a l-a v}$,
TABLE I

PARAMETERS OF THE GENERATION UNITS CONSIDERED IN THE OPTIMIZATION PROBLEM FOR THE CASE STUDY

\begin{tabular}{|c|c|c|c|c|c|}
\hline i & Unit & $C(i, k)$ & $\xi_{g}(i, k)$ & $\hat{d}(i)$ & $P_{g_{\max }(i, k)}$ \\
\hline 1 & $P V$ & 0 & $\xi^{(P V)}(k)$ & 1 & $P_{P V}^{\text {adjusted }}(k)$ \\
2 & $W T$ & 0 & $\xi^{(W T)}(k)$ & 1 & $P_{W T}^{\text {adjusted }}(k)$ \\
3 & $P_{a b s}$ & $C^{(a b s)}(k)$ & 0 & 1 & $P_{\text {abs }}$ \\
4 & $P_{\text {max }}$ & 0 & 0 & -1 & $P_{\text {inj }} j_{\max }$ \\
\hline
\end{tabular}

within uncertainty levels for each time slot. It is known that typical forecast accuracies for systems with low penetration of RES generation can vary dramatically [41]. Hence, the uncertainty levels have been set to $\delta_{1}=10 \%, \delta_{2}=20 \%$, $\delta_{3}=30 \%$ and $\delta_{4}=40 \%$, for each time slot (see Fig. 2).

\section{Optimization}

In the case study, the energy sources are two RESs and the main grid. Particularly, the power exchange of the MG with the main grid, $P_{\text {grid }}(k)$, can be written as the superposition of two auxiliary variables, the absorbed power, $P_{a b s}(k)$, and the injected power, $P_{i n j}(k)$, from/into the main grid as,

$$
P_{\text {grid }}(k)=P_{a b s}(k)-P_{i n j}(k)
$$

Accordingly, Table I shows the variables related to $P_{g}(i, k)$, as well as their associated parameters that have been used in the mathematical formulation. The variables related to the ESS are $P_{E S S}(k)$ and $S o C(k)$.

The optimization problem presented in section II-C is used in the case study for both grid-connected and islanded modes by defining the previously mentioned variables and the parameters presented in Table II. The parameter $P_{R E S}^{\text {adjusted }}(k)$ represents the range in which $P_{P V}^{\text {adjusted }}(k)$ and $P_{W T}^{\text {adjusted }}(k)$ can vary. Some parameters are set to different values in each mode and have been differentiated at the end of the table. In fact, the boundaries of the variables related to the power of the main grid are set to $0 \mathrm{~kW}$ for islanded mode in order to discard this energy source in the optimization problem. In grid-connected mode, these parameters are typically limited by the electrical infrastructure and policies of the utilities. In this case study, $P_{a b s_{\max }}$ has been selected in order to be able to either supply the load if no energy is available in the MG or charge the battery from the main grid, if optimal. Injecting power to the grid is an option if there is RES energy surplus, but it can produce a situation of overvoltage in the distributed system. To limit such events, fixed limitations of the active power feed-in have been mandatory established [40]. In order to model this issue, $P_{i n j_{\max }}$ has been set as the $30 \%$ of the maximum power of each RES.

Regarding the battery, the theoretical operation range of the $S o C$ is from $0 \%$ to $100 \%$. However, it is recommended to establish higher $S o C_{\text {min }}$ since the degradation of the battery is proportional to the Deep of Discharge (DoD) [42]. For the case study in grid-connected mode, the $S o C_{\text {min }}$ has been set by following the recommendation of the IEEE [33], taking advantage of having the grid support. In islanded mode, the ESS is the only grid-forming unit, so it has to operate always in 
TABLE II

Parameters of the Optimization Problem In THE SELECTED MG CASE STUDY

\begin{tabular}{|c|c|c|}
\hline Parameter & \multicolumn{2}{|c|}{ Values and Units } \\
\hline$K$ & \multicolumn{2}{|c|}{$4(\mathrm{~h})$} \\
\hline$\Delta t$ & \multicolumn{2}{|l|}{$1(\mathrm{~h})$} \\
\hline$n_{i}$ & \multicolumn{2}{|l|}{4} \\
\hline$n_{j}$ & \multicolumn{2}{|l|}{1} \\
\hline$P_{R E S}^{\text {adjusted }}(k)$ & \multicolumn{2}{|l|}{$[0-2](\mathrm{kW})$} \\
\hline$P_{L}(k)$ & \multicolumn{2}{|l|}{$0.37(\mathrm{~kW})$} \\
\hline Cap bat $_{\text {b }}$ & \multicolumn{2}{|l|}{$16(\mathrm{kWh})$} \\
\hline$\eta_{\text {bat }}$ & \multicolumn{2}{|l|}{0.95} \\
\hline$P_{E S S_{\max }}(k)$ & \multicolumn{2}{|l|}{$2.2(\mathrm{~kW})$} \\
\hline$P_{E S S_{\min }}(k)$ & \multicolumn{2}{|l|}{$-2.2(\mathrm{~kW})$} \\
\hline$S o C(0)$ & \multicolumn{2}{|c|}{$55-75-95(\%)$} \\
\hline$\xi_{\text {load }}(k)$ & \multicolumn{2}{|l|}{$2(€)$} \\
\hline & Grid-connected mode & Islanded mode \\
\hline$P_{a b s_{\max }}$ & $2 \mathrm{~kW}$ & $0 \mathrm{~kW}$ \\
\hline$P_{i n j_{\max }}$ & $0.6 \mathrm{~kW}$ & $0 \mathrm{~kW}$ \\
\hline$S o C_{\max }$ & $100 \%$ & $90 \%$ \\
\hline$S o C_{\min }$ & $50 \%$ & $20 \%$ \\
\hline$C^{(a b s)}=\xi^{(R E S)}(k)$ & $\begin{array}{c}0.2(€) \forall t \in[6,20](\mathrm{h}) \cup \\
0.1(€) \forall t \notin(6,20)(\mathrm{h})\end{array}$ & $0.2(€)$ \\
\hline
\end{tabular}

voltage control mode. If a full charge of the battery is allowed, the ESS has to change its operation mode to a constant-voltage charge mode (as explained before in section III-A). In this mode, the ESS is not able to continue with the grid forming function. To avoid that the battery reaches the constant-voltage charge mode, it should be operated within a partial state of charge [40], [43]. Therefore, $S o C_{\max }$ is reduced to $90 \%$. Since the ESS in islanded mode cannot be directly controlled, the generation and consumption can be adjusted by the EMS-MG in order to keep the battery within a partial SoC. Meanwhile, $S o C_{\min }$ is also reduced so that the battery can be more discharged and the load can be longer supplied.

The unitary cost of buying energy to the main grid is defined by the distribution system operator. In the case study, the tariff is established in a Time of Use (ToU) scheme in which the cost is higher during the day $(t \in\{6,20\}$ (h)). Moreover, the penalization coefficients $\xi^{(P V)}$ and $\xi^{(W T)}\left(\xi^{(R E S)}\right.$ are set as the unitary cost of buying energy from the grid $C^{(a b s)}$ since the free energy that is not used should be bought later on at this cost. The penalization cost related to the load, $\xi_{\text {load }}$, is selected to be one order of magnitude greater than $C^{(a b s)}$ in order to prioritize the comfort of the user over the operating cost. Since these parameters are defined in the continuous framework (in terms of $t$ ), the Processor has to read the related data for next time window and transform them into the suitable discrete format to be used by the Optimization Process.

\section{EXPERIMENTAL RESULTS}

In order to evaluate the proposed online MG-EMS, the case study presented in the previous section is implemented experimentally in the Microgrid Research Laboratory of the Aalborg University [44]. Along this section, we present the experimental setup, the operation of the system and the validation of the MG-EMS under a proposed evaluation framework.

\section{A. Experimental Implementation}

The whole system is tested experimentally in a laboratory scale microgrid under an architecture with three levels: software level, real time platform and physical level, as shown in Fig. 5. The controllers run in real time but the time of generation profiles and scheduling are scaled down from hours to minutes (1 h:1 min), as in [19], [45].

In the software level, the online MG-EMS has been implemented mainly in Labview, except for the Optimization Problem module. It has been developed in an Algebraic Modeling Language (AML) called GAMS with the solver CPLEX to compute the problem [46]. This program is called by LabView through the tool Matlab Script. Moreover, the communication between the MG-EMS and the real-time platform is established by means of a User Datagram Protocol (UDP) over Ethernet, while the sampling time of the $D A Q$ \& $H M I$ module is set to $1 \mathrm{~ms}$. The update time of the control references is $t_{r e p}=15 \min (15 \mathrm{~min}: 15 \mathrm{~s})$ and each time slot has a duration of $\Delta t=1 \mathrm{~h}(1 \mathrm{~h}: 1 \mathrm{~min})$.

The real-time platform includes the controllers and models of the energy resources connected to the grid-side converters. In the case of the battery, a detailed electrical model of a valveregulated lead-acid (VRLA) is applied as previously presented and validated in [47], [48]. Meanwhile, the RESs modeled as power sources in accordance with PV and WT generation profiles derived from real data, obtained from [49].

The physical level is a real electrical network integrated by the grid side inverters and their corresponding LCL filters. The inverters are fed by stiff DC source, what allows to control and operate them in accordance with the models and generation profiles of their corresponding DER. To the common bus are also connected the transformer to the main grid and a resistive load of $380 \mathrm{~kW}$. The experimental setup is shown in Fig. 6

\section{B. Operation of the MG-EMS}

The performance of the system with the proposed MGEMS in grid-connected and islanded modes is presented by comparing with the results obtained in autonomous operation.

1) Grid-connected Mode: In the case of grid-connected mode, the online MG-EMS is compared with an enhanced autonomous case. Considering the ToU scheme, the battery is charged with constant power reference at the beginning of the day, when the grid cost is lower (Night Price, N.P.) and then, the battery power follows the unbalance between generation and demand. The value to charge the battery has been set to $300 \mathrm{~W}$, by assuming the charging criteria of $0.3 C_{20}$ [33].

The experimental results are presented in Fig. 7 for autonomous operation (a) and online MG-EMS strategy (b). As can be seen, the battery is charged by energy absorbed from the grid $(S 1)$ during more time in the autonomous mode than in online scheduling case, since the last one can anticipate when the price of the grid will increase in the time horizon, $K$, and thus, estimate how much the battery should be charged to supply the load in a cheaper way.

In this generation scenario, the battery reaches its maximum level of charge and the ESS changes its control to the ConstantVoltage charge mode ( $S 3$ in Fig. 7, when $V_{b a t}$ reaches $V_{t h}$ ). 
Real Time Platform

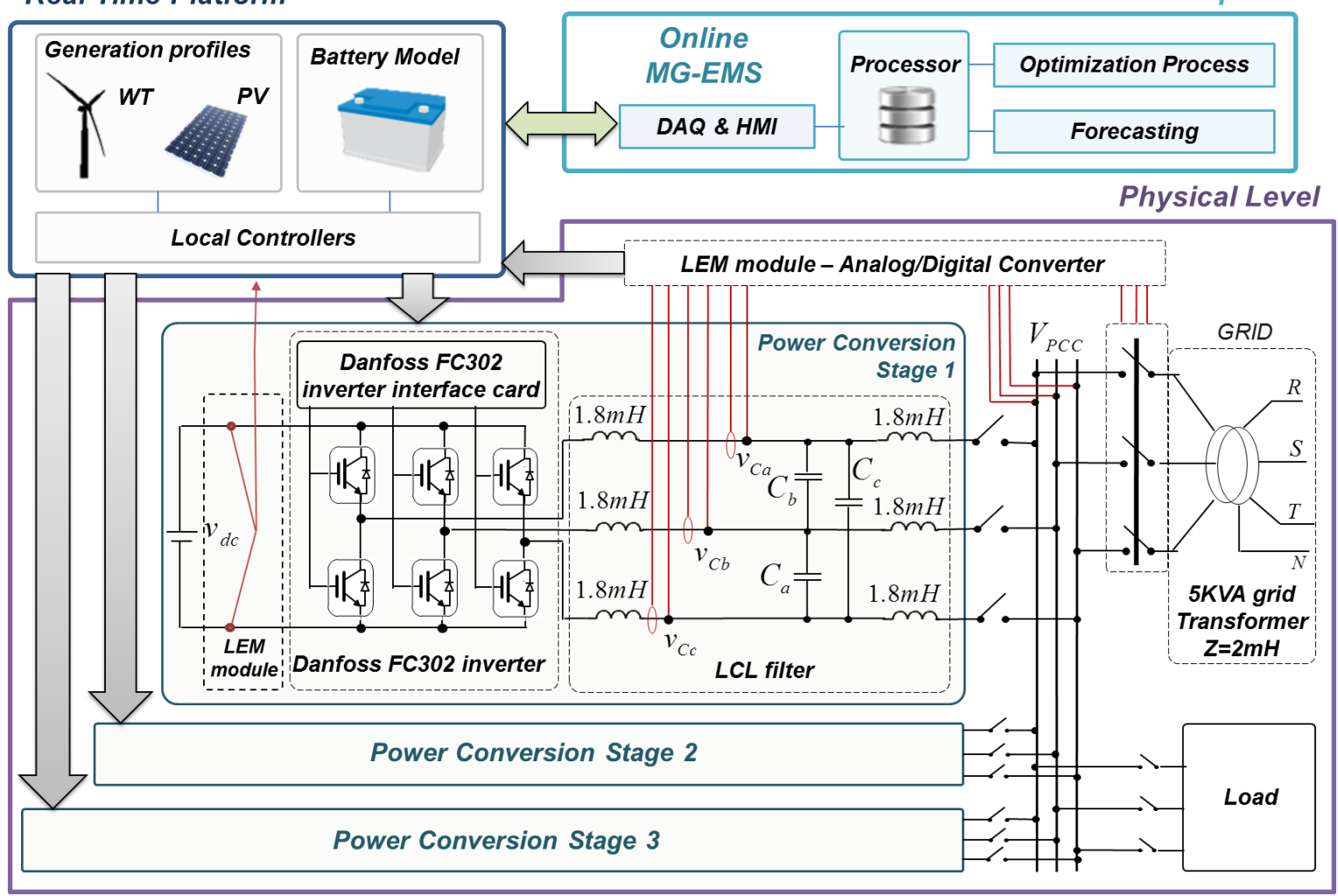

Fig. 5. Levels of the architecture implemented experimentally

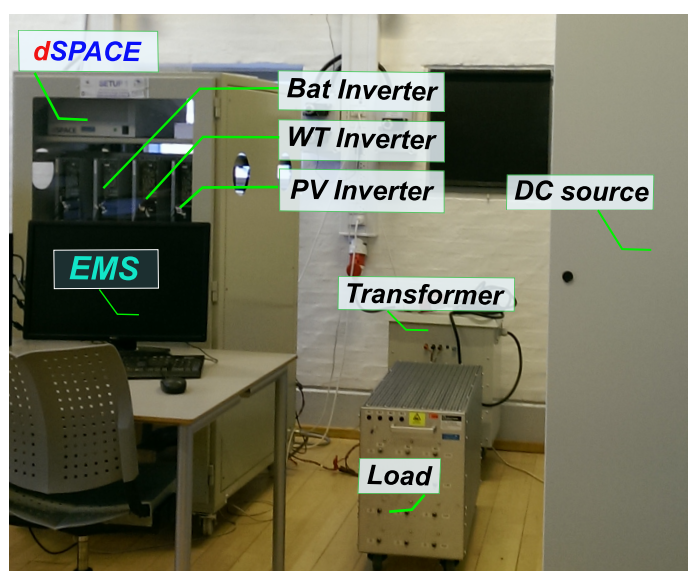

Fig. 6. Experimental setup

This action occurs earlier in the autonomous mode than in the online schedule case. Accordingly, in the first case, there is more power curtailment because the battery is not able to store surplus energy. Moreover, since the process of charging a battery is typically inefficient, it is necessary to return it more than a $100 \%$ of the energy during the saturation stage [42]. Typical values of overcharge can range from a $5 \%$ to a $30 \%$ [33]. This overcharge can be evidenced at $t 1$ in Fig. 7.

The objective function evaluated in autonomous operation is $0,32 €$ while in optimized operation is $0,177 €$. Since the system relies on the power provided by the main grid to supply the load, there does not cost related to penalization for its disconnection at any time, i.e. $J_{3}=0$ due to $z_{\text {load }}(k)=$ $1, \forall k \in K$ within each $T$ in both cases. The results show that the performance of the MG with the proposed online MG-EMS is improved based on the defined objective function. Moreover, the accumulated costs of $\mathrm{J} 1$ and $\mathrm{J} 2$ over time for the two approaches are presented in Fig. 8. It can be seen that the online MG-EMS reduces the costs associated with the energy absorbed from the grid, but also it allows the MG to take more advantage of the RES energy.

2) Islanded Mode: In this mode, the battery works as the single grid-forming unit and, consequently, it cannot be managed by the MG-EMS. Accordingly, MG-EMS just provides the power references for RESs and the commands for dis/connecting the load. The operation of the MG working with the MG-EMS is compared with the system working autonomously. In the last case, RESs follow MPPT references and the system relies on the contingency actions explained in section III-A in order to avoid damages of the ESS.

The experimental results in both cases are presented in Fig. 9 and their associated $J(X, Z)$ are $1.135 €$ and $0.88 €$, in autonomous and optimized operation, respectively. Since RES generation during the day is enough to not disconnect the load at any time, the numerical results correspond just to the penalization curtailment of the RESs, $J 2(X, Z)$, and they show that the RES energy limited in autonomous operation is larger than by implementing the MG-EMS. The limitation of the RES power is performed mainly to the WT with the proposed MG-EMS ( $P 2$ in Fig. 9 (a)) while in the other case, both generation units should be set to $0 \mathrm{~kW}$, one by one when 

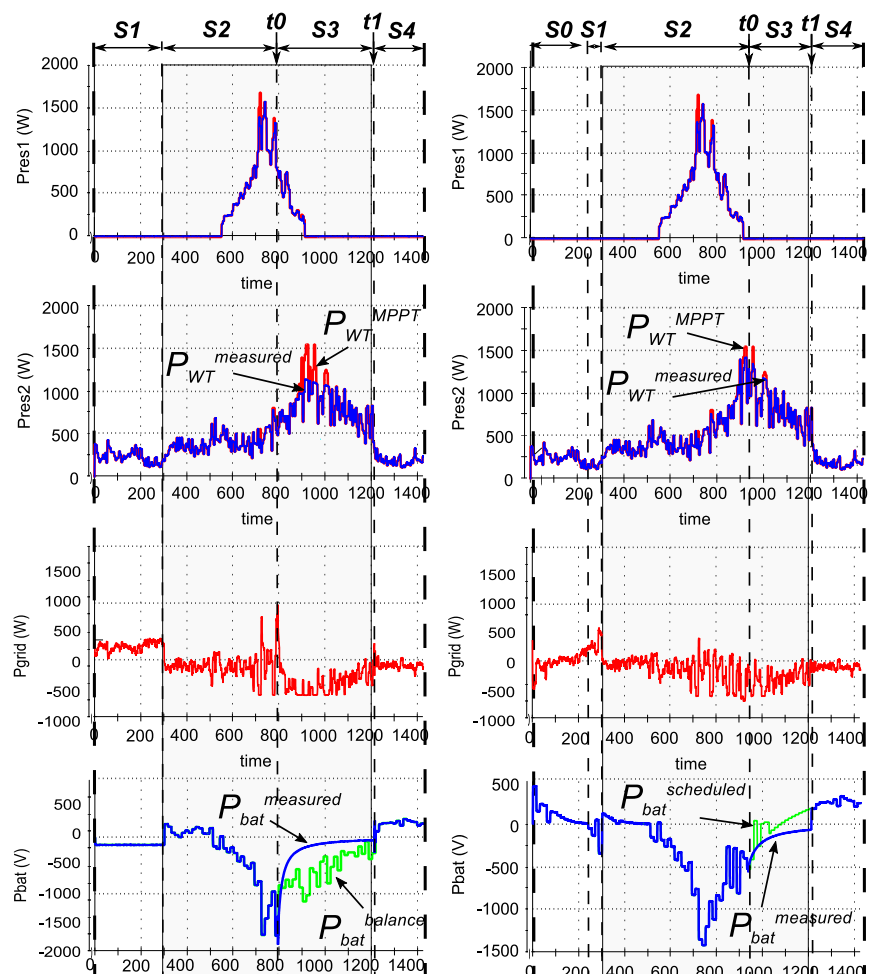

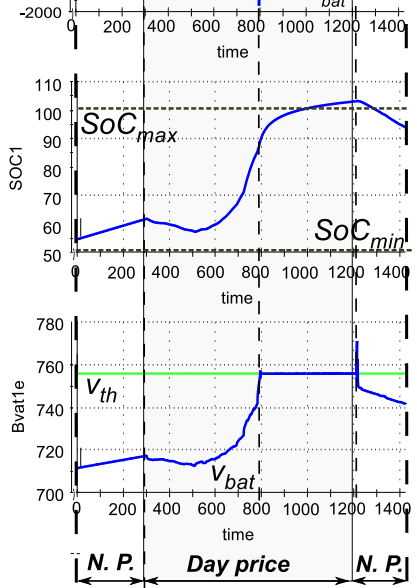

(a)
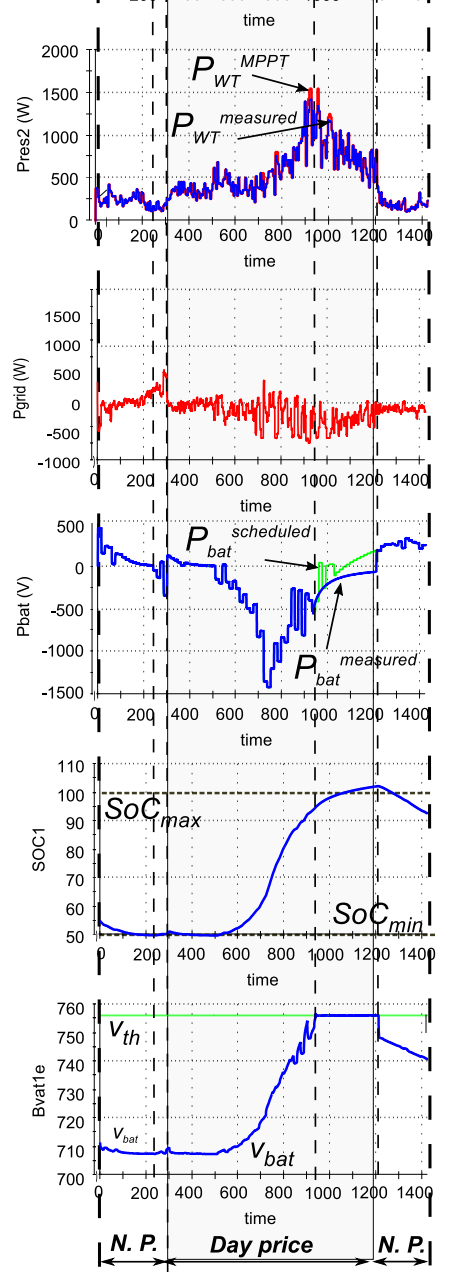

(b)
Fig. 7. Experimental results for the microgrid in grid-connected mode with (a) autonomous and (b) optimized scheduling. Top to bottom: PV power, WT power, power of the main grid, battery power, SoC of the battery, Battery voltage.

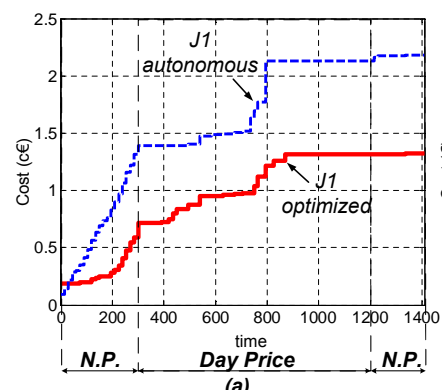

(a)

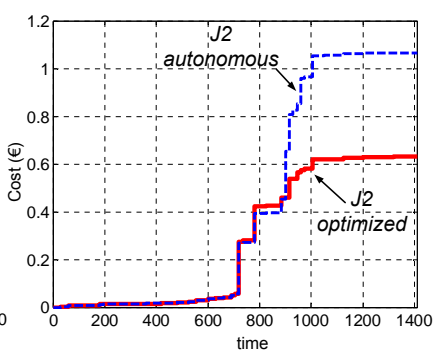

(b)
Fig. 8. Costs comparison between experimental results with the autonomous operation (dashed lines) and optimized scheduling (solid lines). Accumulated costs associated with (a) grid costs, $J 1$ and (b) RES curtailment, J2

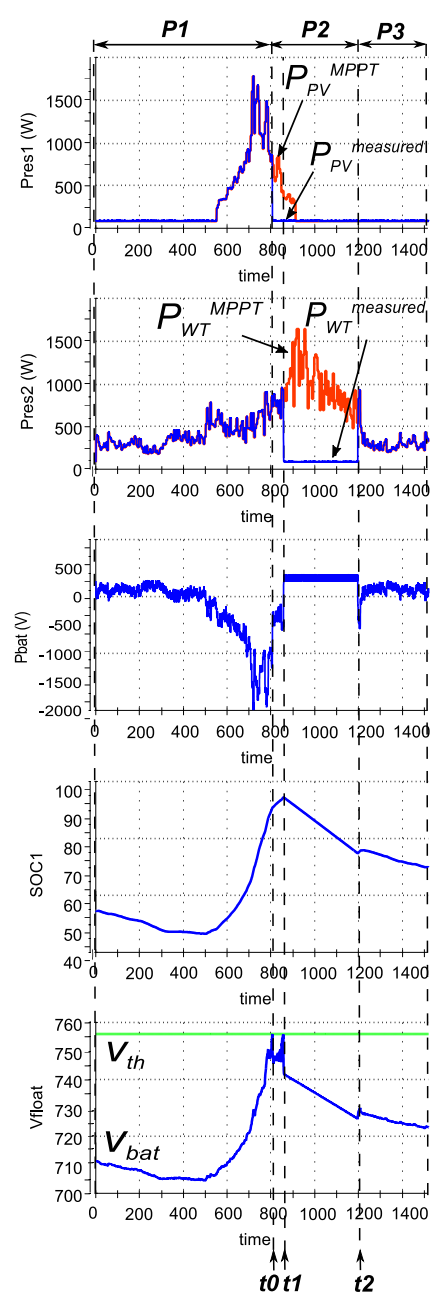

(a)
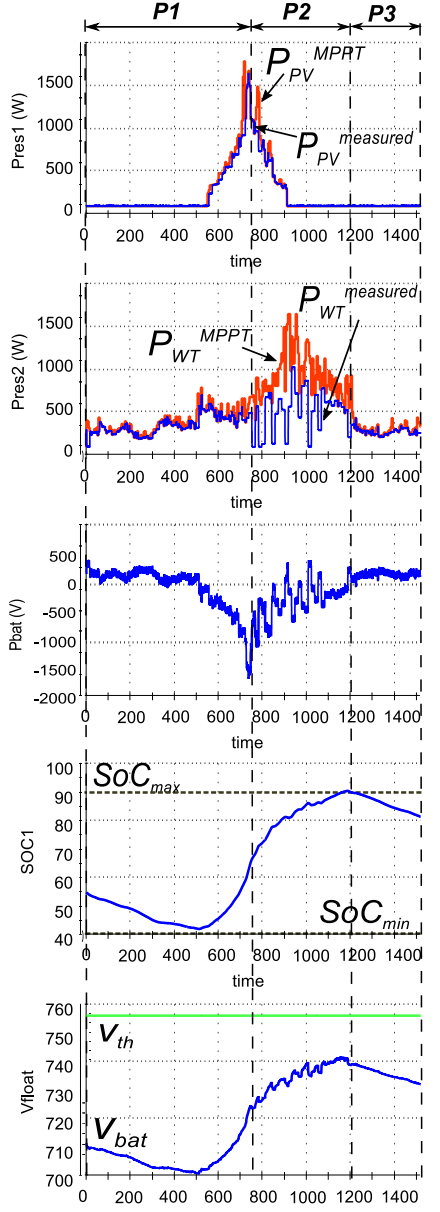

time

(b)
Fig. 9. Experimental results in islanded mode with (a) autonomous and (b) optimized scheduling. Top to bottom: PV power, WT power, battery power, SoC of the battery, Battery voltage.

$v_{b a t}=v_{t h}(P 2$ in Fig. 9 (b)). In fact, with the proposed MGEMS, the risk of damage for overvoltage is reduced since the generation is been controlled all the time. In this way, the amount of energy stored in the battery is managed indirectly.

3) Operation under load disconnections and large generation uncertainties: In order to test the proposed MG-EMS under abnormal situations, two scenarios have been defined in islanded mode. The first scenario shows how the system works when the load is suddenly disconnected but still the MGEMS can detect the power requirements. The second scenario presents how the system reacts when an unexpected outage of WT generation occurs. In this case, the MG-EMS does not detect the outage and continues using the WT power adjusted data. The experimental results are shown in Fig. 10.

Under the first scenario (Fig. 10 (a)), the non-scheduled load disconnection occurs from $t 0$ until $t 1$. Since the MGEMS detects the fault, the optimization problem assumes that $P_{L}=0 \mathrm{~W}$. In this way, the references provided by the MGEMS are adjusted to the new situation and the whole system works properly. During the load disconnection, the battery is charged without limiting the RES energy while its SoC is 

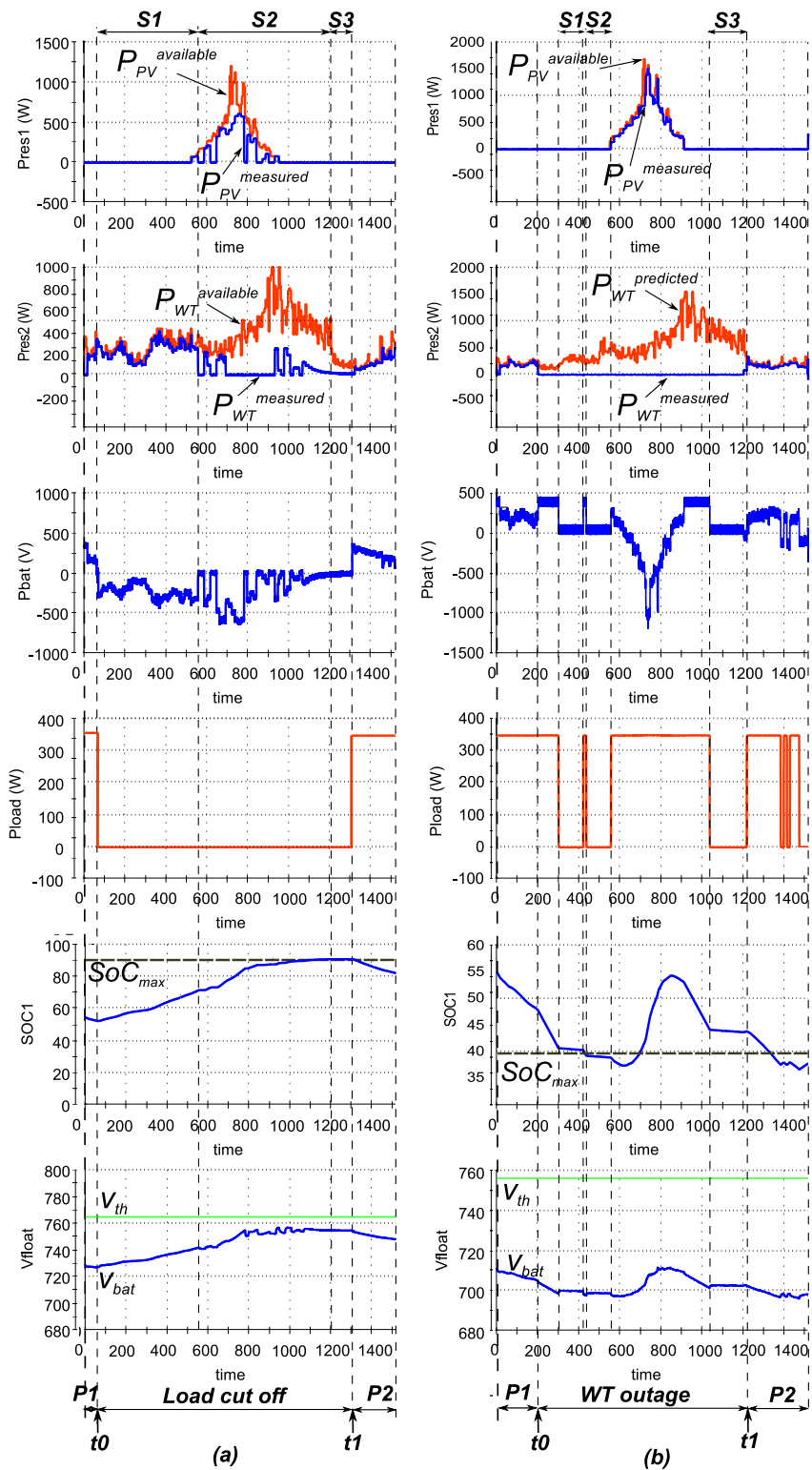

Fig. 10. Experimental results for islanded mode under (a) unexpected load disconnection and (b) unexpected outage of WT generation. Top to bottom: PV power, WT power, battery power, load power, SoC of the battery, battery voltage.

low enough $(S 1)$. After that, part of the RES is limited to reach the upper boundary of the SoC, $S o C_{\max }$, in a smooth way $(S 2)$. Once the SoC gets the value of $S o C_{\max }$, the RES generation is set to $0 \mathrm{~W}(S 3)$. These references are kept until the reconnection of the load $(P 2)$. At this moment, the value of $P_{L}$ is re-established and accordingly, RES energy is not limited and the battery is discharged to manage the differences between generation and demand.

In the second scenario shown in Fig. 10(b), the WT power is set to $0 \mathrm{~kW}$ from $t 0$ until $t 1$ to test the system under a high mismatch between the forecast and available generation. Despite the forecast profile is adjusted by means of the measurements as mentioned in section II, the obtained predicted profile retains the tendency of the curve, what results in additional unbalances that should be managed by the battery. Since, in this case, the system has a low generation, the MG-
EMS should schedule the disconnection of the load. During the WT outage, the load is disconnected during $S 1, S 2$ and $S 3$. As can be seen, the system intends to keep connected the load even when there is not enough energy in the ESS (when $S o C<S o C_{m i n}$ ), since it relies on the WT generation. Before $S 1$, there is not available RESs energy and accordingly, the battery is the responsible for supplying the load until the MG-EMS does not foresee energy enough to fed the demand neither from RES nor the battery and disconnects the load (S1). During $S 2$, the optimization problem results to be infeasible because the minimum boundaries of $S o C$ cannot be achieved according to the adjusted forecast data. Therefore, the MG-EMS sets $z_{\text {load }}=0$ and not limited the RES generation in order to allow the battery to be charged during the intervals of an infeasible condition.

After $S 2$, the MG-EMS foresees to have some energy from RESs to supply the load but $S o C<S o C_{\min }$ in some intervals, because of the low $\mathrm{SoC}$ conditions and the big prediction mismatch. During $S 3$, the system anticipates that available energy will decrease and thus, there will not be energy enough to supply the load and increase the level of $\mathrm{SoC}$, and thus, disconnects the load. When finally the WT is putting into operation $(P 2)$, the system starts to schedule the commands according to more accurate adjusted forecast data. However, it can be seen that in normal operation, when $S o C$ is near $S o C_{m i n}$, the MG-EMS can schedule successive connection/disconnection cycles, due to the updated forecasting.

As can be seen, even under the critical situation of WT generation outage during almost one day, the minimum value of $\mathrm{SoC}$, in this case, is infringed in just $7.5 \%$.

\section{Evaluation Framework}

In order to validate the proposed online MG-EMS, an evaluation framework is defined in which the performance of the MG-EMS is compared experimentally with a conventional approach, but also with reference strategies that define upper and lower bounds. This framework allows having a better understanding of how good is the proposal related to the worst case and how much the strategy can still be improved by comparing to the best-known case.

Henceforth, the conventional strategy will be notated as EMS1, the proposed online MG-EMS as EMS2 and the reference cases as Worst Case (upper bound) and Best Case (lower bound), respectively. The defined strategies are summarized in Table III. The case EMS2pf corresponds to the proposed online MG-EMS tested with perfect average weather forecast so that the influence of the uncertainties over the operating cost can be appreciated. Both, EMS2 and EMS2pf, are implemented with $\Delta t=1 \mathrm{~h}$ and $t_{r e p}=15 \mathrm{~min}$.

This evaluation framework has been applied in the selected case study in grid-connected mode, which is the normal operation mode of the microgrid and provides more degrees of freedom to the optimization since the battery can be managed. The performance of every strategy is compared by evaluating the operating costs (objective function value, $J(X, Z)$ in (3)).

In light of the above, the Worst Case is defined as a reference for the situation of autonomous operation of the 
TABLE III

ONLINE EMS STRATEGIES USED IN QUANTITATIVE ASSESSMENT

\begin{tabular}{|c|c|}
\hline Strategy & Description \\
\hline Worst Case & Without battery \\
EMS1 & Conventional battery management \\
EMS2 & Proposed EMS with forecast errors \\
EMS2pf & Proposed EMS without forecast errors \\
Best Case & EMS with complete and perfect information \\
\hline
\end{tabular}

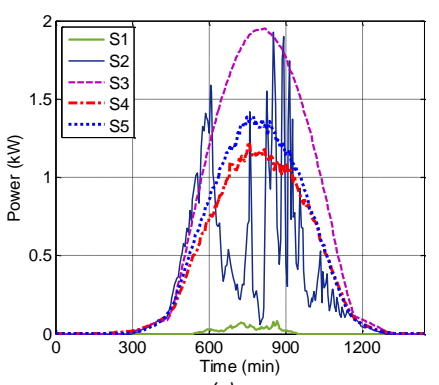

(a)

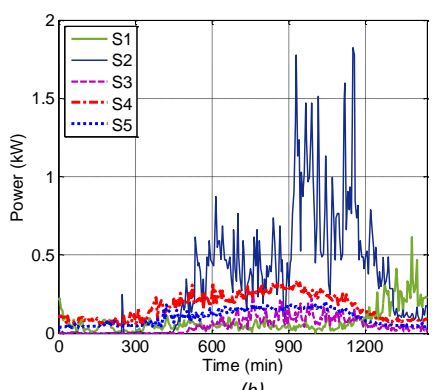

(b)
Fig. 11. Generation profiles used in the evaluation assessment. (a) PV power, (b) WT power

microgrid without using the battery. In turn, the Best Case is defined as a reference for the situation in which the management is performed by reducing the update time of the optimization $\left(t_{r e p}=2 \mathrm{~min}\right)$ and using complete and perfect information. The complete information is related to foresee the data of the whole day and not only $4 \mathrm{~h}$ ahead, while the perfect information is related to know exactly the generation profiles with a short resolution $(\Delta t=5 \mathrm{~min})$. Accordingly, every time slot for the scheduling process is set to $5 \mathrm{~min}$ rather than $1 \mathrm{~h}$. In this ideal case, the MG-EMS can provide the economically optimal references by knowing exactly the generation profiles and the ToU scheme of the whole day. In addition, since the updating of data is faster, many unexpected changes (for example, due to battery dynamics) can be observed and decisions can be quickly adapted to minimize costs. The strategy EMS1 manages the battery with a conventional charge strategy, in which the battery power follows the average unbalance between the generation and demand [40]. The power battery reference is updated every $t_{r e p}=15 \mathrm{~min}$, as in the online proposed strategy.

In order to address a quantitative analysis of the proposal, fifteen scenarios are set by selecting five different generation profiles and considering three initial conditions of SoC. The selected power profiles are notated as S1 to S5, as presented in Fig. 11. Likewise, three initial conditions of $S o C$ are set, $55 \%, 75 \%$ and $95 \%$. In this way, the scenarios used in this framework are defined and denoted by using each set of generation profiles with every initial condition (e.g. using S1 with $\operatorname{SoC}(0)=55 \%$, the scenario is denoted as S1-55).

The results, summarized in Table IV and graphically presented in Fig. 12, show the improvement produced by management and the differences reached by different EMS, on the considered scenarios. The Worst Case is improved by all the defined strategies, with gaps depending on the RES power generation and the energy available in the ESS. Comparing to
TABLE IV

OBJECTIVE FUNCTION RESULTS OBTAINED EXPERIMENTALLY

\begin{tabular}{|c|c|c|c|c|c|}
\hline & \multicolumn{5}{|c|}{ Daily Objective Cost $(€)$} \\
\hline Scenario & Worst Case & EMS1 & EMS2 & EMS2pf & Best Case \\
\hline S1-55 & 1,107 & 1,012 & 0,957 & 0,936 & 0,633 \\
S1-75 & 1,107 & 0,752 & 0,751 & 0,735 & 0,454 \\
S1-95 & 1,107 & 0,455 & 0,433 & 0,455 & 0,275 \\
S2-55 & 3,063 & 0,922 & 0,696 & 0,599 & 0,558 \\
S2-75 & 3,063 & 0,714 & 0,557 & 0,445 & 0,362 \\
S2-95 & 3,063 & 0,611 & 0,685 & 0,481 & 0,369 \\
S3-55 & 4,241 & 0,914 & 0,731 & 0,641 & 0,502 \\
S3-75 & 4,241 & 0,670 & 0,540 & 0,472 & 0,274 \\
S3-95 & 4,241 & 0,763 & 0,665 & 0,584 & 0,416 \\
S4-55 & 1.596 & 0.252 & 0.239 & 0.223 & 0.196 \\
S4-75 & 1.596 & 0.095 & 0.064 & 0.062 & 0.056 \\
S4-95 & 1.596 & 0.042 & 0.041 & 0.041 & 0.019 \\
S5-55 & 1.722 & 0.291 & 0.244 & 0.249 & 0.239 \\
S5-75 & 1.722 & 0.130 & 0.051 & 0.051 & 0.045 \\
S5-95 & 1.722 & 0.052 & 0.042 & 0.024 & 0.021 \\
\hline
\end{tabular}

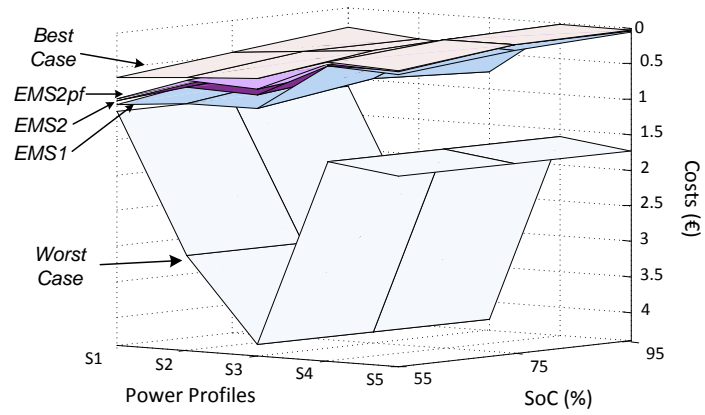

Fig. 12. Operational costs for the different scenarios defined for the evaluation assessment

the Best Case, the gaps with the strategies are shorter but still, there is some improvement that can be achieved. In general, the cost obtained with each EMS in the defined scenarios is higher when the initial condition of SoC is low because the RES generation at the beginning of the day is also low and therefore, it is needed to absorb power from the main grid. On the other hand, the lowest costs are incurred when the RES generation and the stored energy available are enough to supply the load without having to curtail RES power.

The proposed MG-EMS without considering uncertainties (EMS2pf) is shown to be better than EMS1 in all cases, except for scenario S1-95, in which the operational cost is the same for both strategies. In this case, the system foresees in each iteration that the best decision is to fulfill the unbalance power between demand and generation, given the time horizon, which is the basis of EMS1.

Considering the proposed MG-EMS with forecast errors (EMS2), this is better than EMS1 and worse than EMS2pf, most of the cases. Occasionally, this tendency can change, as in S1-95, S3-95 and S5-55. This is because the decisions made by EMS2 in one iteration, which are affected by errors due to the prediction of the power generation, influence the condition of the next iterations, improving or degrading the total operating costs. 


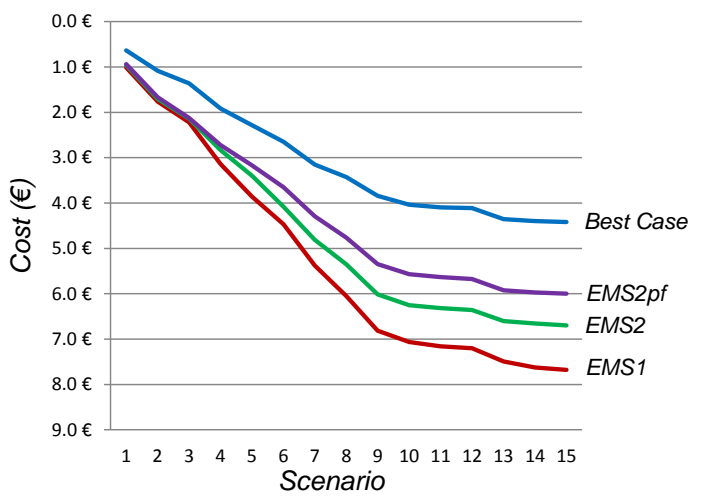

Fig. 13. Cumulative operational costs extrapolated from the defined scenarios

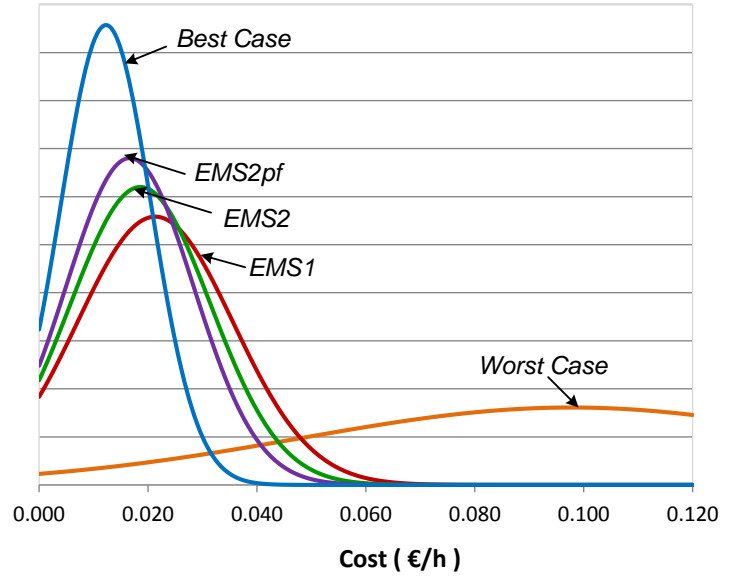

Fig. 14. Probability distribution of cost for each EMS

In order to better display the difference between the EMSs (without including the Worst Case) and highlight the long-term effects on savings, cumulative costs for all the scenarios are presented in Fig. 13. The trends show that EMS1 is surpassed at long term by EMS2, even with forecast errors. In addition, they illustrate that the performance of EMS2 can be improved by using more accurate forecasting module (EMSpf).

For comparative purposes, the costs obtained for the $24 \mathrm{~h}$ horizon are expressed in terms of hourly costs. Accordingly, the average cost with $95 \%$ confidence is given in Table V. In order to illustrate and further discuss the results in Table V, the probability distribution of costs and savings are shown in Figs. 14 and 15 . The deviation of the data is related to the diversity of the selected input data. From Fig. 14, it is evident that probability shifts towards greater savings when more sophisticated energy management systems are implemented. The distance between the proposed EMS2 and the best case shows that more work still needs to be performed in this field. Again, the evaluation framework allows comparing different EMS strategies and demonstrates that the probability of getting lower costs increases by improving the management methods. Finally, it is worth noting the non-null probability of reaching a zero cost operation, which indicates that for certain scenarios a conveniently operated micro-grid may not require the purchase of energy from the grid.

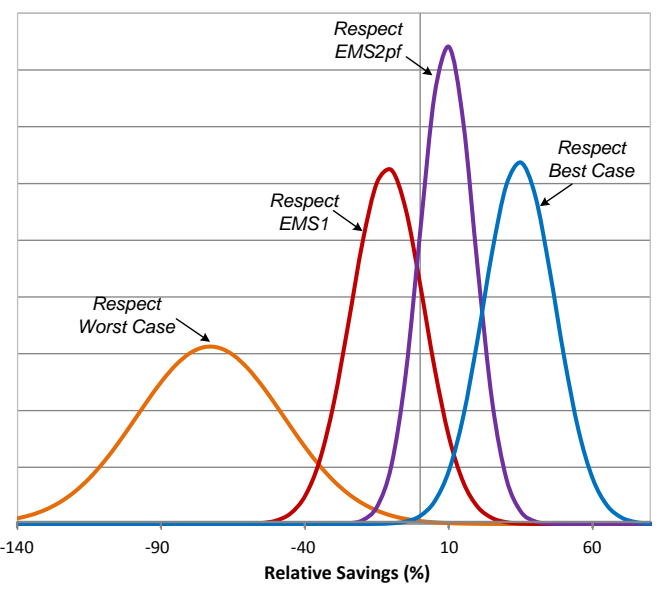

Fig. 15. Probability distribution of the EMS2 respect to the other strategies

As can be seen from Fig. 15, the proposed EMS2 produces average savings of $81,0 \%$ respect to the Worst Case. The proposed EMS2 performs better than EMS1 with an average operational savings of $12,7 \%$. Meanwhile, the gap between EMS2 and EMSpf shows that still the strategy can be improved by an average of $10,5 \%$, with the same considerations, i.e. time horizons, $\Delta t$ and time slots by improving forecasting methods. In the same way, by comparing EMS2pf to EMS1, the average savings can reach to $21,9 \%$. However, EMS2 is shown to be still $34 \%$ average higher than the Best Case, which is the gap that could be also attained if complete and perfect information was available.

\section{CONCLUSIONS}

In this paper, an adaptable online MG-EMS has been designed and experimentally tested in order to deal with the variability and uncertainty feature of microgrid systems with RESs. The presented MG-EMS architecture manages to get a proper integration and interaction between modules as well as the interface with controller level. In turn, the online scheme dynamically adjusts the forecast data by considering the current measured and consequently, the optimization process can select more convenient set points related to the defined objective function. Within the optimization module, an economic optimization has been defined in a generic way for generation-side management, effective for grid-connected and islanded modes. This proposal has been experimentally tested and demonstrated its promising capability to work under different operational modes of the microgrid, reducing the operating cost. A quantitative evaluation framework has been proposed and used in the case study, demonstrating the effectiveness of the proposal over a selected benchmark strategy and establishing the gaps with ideal boundaries of the best and worst possible solutions. This also establishes a reference for future improvements that may be attained with further refinements of the management strategy. As future work, the proposed architecture can be improved by implementing sophisticated forecasting methods, testing other optimization models or adding functionalities, such as diagnostics or power quality modules. 
TABLE V

Statistical Results from Average Operational Costs IN $€ / h$

\begin{tabular}{|c|c|c|c|c|c|}
\hline & Worst Case & EMS1 & EMS2 & EMS2pf & Best Case \\
\hline Average $(€ / \mathrm{h})$ & 0.098 & 0.021 & 0.019 & 0.017 & 0.012 \\
\hline Standard Dev $(€ / \mathrm{h})$ & 0.050 & 0.014 & 0.013 & 0.012 & 0.008 \\
\hline Confidence Interval $(€ / \mathrm{h})$ & 0.025 & 0.007 & 0.007 & 0.006 & 0.004 \\
\hline Performance $(€ / \mathrm{h})$ & $\begin{array}{c}0,1 \pm 0,03 \\
( \pm 25,6 \%)\end{array}$ & $\begin{array}{c}0,021 \pm 0,007 \\
( \pm 33,9 \%)\end{array}$ & $\begin{array}{c}0,019 \pm 0,007 \\
( \pm 35 \%)\end{array}$ & $\begin{array}{c}0,017 \pm 0,006 \\
( \pm 35,6 \%)\end{array}$ & $\begin{array}{c}0,012 \pm 0,004 \\
( \pm 34,4 \%)\end{array}$ \\
\hline
\end{tabular}

\section{REFERENCES}

[1] J. Guerrero, J. Vsquez, J. Matas, L. de Vicuña, and M. Castilla, "Hierarchical control of droop-controlled $\{\mathrm{AC}\}$ and $\{\mathrm{DC}\}$ microgrids - a general approach toward standardization," IEEE Transactions on Industrial Electronics, vol. 58, no. 1, pp. 158-172, Jan. 2011.

[2] J. Vasquez, J. Guerrero, M. Savaghebi, J. Eloy-Garcia, and R. Teodorescu, "Modeling, analysis, and design of stationary-reference-frame droop-controlled parallel three-phase voltage source inverters," IEEE Transactions on Industrial Electronics, vol. 60, no. 4, pp. 1271-1280, April 2013.

[3] F. Nejabatkhah and Y. W. Li, "Overview of power management strategies of hybrid ac/dc microgrid," IEEE Transactions on Power Electronics, vol. 30, no. 12, pp. 7072-7089, Dec 2015 .

[4] H. Zhou, T. Bhattacharya, D. Tran, T. S. T. Siew, and A. M. Khambadkone, "Composite energy storage system involving battery and ultracapacitor with dynamic energy management in microgrid applications," IEEE Transactions on Power Electronics, vol. 26, no. 3, pp. 923-930, March 2011

[5] N. L. Diaz, J. G. Guarnizo, M. Mellado, J. C. Vasquez, and J. M. Guerrero, "A robot-soccer-coordination inspired control architecture applied to islanded microgrids," IEEE Transactions on Power Electronics, vol. PP, no. 99, pp. 1-1, 2016.

[6] D. Saez, F. Avila, D. Olivares, C. Canizares, and L. Marn, "Fuzzy prediction interval models for forecasting renewable resources and loads in microgrids," IEEE Transactions on Smart Grid, vol. 6, no. 2, pp. 548556, March 2015.

[7] N. L. Diaz, A. C. Luna, J. C. Vasquez, and J. M. Guerrero, "Centralized control architecture for coordination of distributed renewable generation and energy storage in islanded ac microgrids," IEEE Transactions on Power Electronics, vol. PP, no. 99, pp. 1-1, 2016.

[8] J. de Matos, F. S.F.e Silva, and L. de S Ribeiro, "Power control in ac isolated microgrids with renewable energy sources and energy storage systems," IEEE Transactions on Industrial Electronics, vol. 62, no. 6, pp. 3490-3498, June 2015

[9] H. Mahmood, D. Michaelson, and J. Jiang, "Accurate reactive power sharing in an islanded microgrid using adaptive virtual impedances," IEEE Transactions on Power Electronics, vol. 30, no. 3, pp. 1605-1617, March 2015

[10] Y. Han, H. Li, P. Shen, E. A. A. Coelho, and J. M. Guerrero, "Review of active and reactive power sharing strategies in hierarchical controlled microgrids," IEEE Transactions on Power Electronics, vol. 32, no. 3 , pp. 2427-2451, March 2017.

[11] V. Kekatos, G. Wang, A. J. Conejo, and G. B. Giannakis, "Stochastic reactive power management in microgrids with renewables," IEEE Transactions on Power Systems, vol. 30, no. 6, pp. 3386-3395, Nov 2015.

[12] C. A. Hernandez-Aramburo, T. C. Green, and N. Mugniot, "Fuel consumption minimization of a microgrid," IEEE Transactions on Industry Applications, vol. 41, no. 3, pp. 673-681, May 2005.

[13] I. U. Nutkani, P. C. Loh, and F. Blaabjerg, "Droop scheme with consideration of operating costs," IEEE Transactions on Power Electronics, vol. 29, no. 3, pp. 1047-1052, March 2014.

[14] A. Elrayyah, F. Cingoz, and Y. Sozer, "Construction of nonlinear droop relations to optimize islanded microgrid operation," IEEE Transactions on Industry Applications, vol. 51, no. 4, pp. 3404-3413, July 2015.

[15] F. Cingoz, A. Elrayyah, and Y. Sozer, "Plug-and-play nonlinear droop construction scheme to optimize islanded microgrid operations," IEEE Transactions on Power Electronics, vol. 32, no. 4, pp. 2743-2756, April 2017.

[16] I. U. Nutkani, P. C. Loh, P. Wang, and F. Blaabjerg, "Cost-prioritized droop schemes for autonomous ac microgrids," IEEE Transactions on Power Electronics, vol. 30, no. 2, pp. 1109-1119, Feb 2015.
[17] M. Soroush and D. J. Chmielewski, "Process systems opportunities in power generation, storage and distribution," Computers \& Chemical Engineering, vol. 51, pp. 86 - 95, 2013.

[18] F. Katiraei, R. Iravani, N. Hatziargyriou, and A. Dimeas, "Microgrids management," Power and Energy Magazine, IEEE, vol. 6, no. 3, pp. 54-65, May 2008.

[19] A. C. Luna, N. L. D. Aldana, M. Graells, J. C. Vasquez, and J. M. Guerrero, "Mixed-integer-linear-programming based energy management system for hybrid pv-wind-battery microgrids: Modeling, design and experimental verification," IEEE Transactions on Power Electronics, vol. PP, no. 99 , pp. $1-1,2016$

[20] A. Soroudi and T. Amraee, "Decision making under uncertainty in energy systems: State of the art," Renewable and Sustainable Energy Reviews, vol. 28, pp. 376 - 384, 2013.

[21] L. Meng, E. R. Sanseverino, A. Luna, T. Dragicevic, J. C. Vasquez, and J. M. Guerrero, "Microgrid supervisory controllers and energy management systems: A literature review," Renewable and Sustainable Energy Reviews, vol. 60, pp. 1263 - 1273, 2016.

[22] P. T. Baboli, M. Shahparasti, M. P. Moghaddam, M. R. Haghifam, and M. Mohamadian, "Energy management and operation modelling of hybrid ac-dc microgrid," IET Generation, Transmission Distribution, vol. 8, no. 10, pp. 1700-1711, October 2014.

[23] H. Kakigano, Y. Miura, and T. Ise, "Distribution voltage control for dc microgrids using fuzzy control and gain-scheduling technique," IEEE Transactions on Power Electronics, vol. 28, no. 5, pp. 2246-2258, May 2013.

[24] C. Li, F. Bosio, F. Chen, S. K. Chaudhary, J. Vasquez, and J. Guerrero, "Economic dispatch for operating cost minimization under real time pricing in droop controlled dc microgrid," IEEE Journal of Emerging and Selected Topics in Power Electronics, vol. PP, no. 99, pp. 1-1, 2016.

[25] C. Chen, S. Duan, T. Cai, B. Liu, and G. Hu, "Smart energy management system for optimal microgrid economic operation," IET Renewable Power Generation, vol. 5, no. 3, pp. 258-267, May 2011.

[26] G. Oriti, A. L. Julian, and N. J. Peck, "Power-electronics-based energy management system with storage," IEEE Transactions on Power Electronics, vol. 31, no. 1, pp. 452-460, Jan 2016.

[27] Y. K. Chen, Y. C. Wu, C. C. Song, and Y. S. Chen, "Design and implementation of energy management system with fuzzy control for dc microgrid systems," IEEE Transactions on Power Electronics, vol. 28, no. 4, pp. 1563-1570, April 2013.

[28] E. Barklund, N. Pogaku, M. Prodanovic, C. Hernandez-Aramburo, and T. C. Green, "Energy management in autonomous microgrid using stability-constrained droop control of inverters," IEEE Transactions on Power Electronics, vol. 23, no. 5, pp. 2346-2352, Sept 2008.

[29] S. A. Pourmousavi, M. H. Nehrir, C. M. Colson, and C. Wang, "Realtime energy management of a stand-alone hybrid wind-microturbine energy system using particle swarm optimization," IEEE Transactions on Sustainable Energy, vol. 1, no. 3, pp. 193-201, Oct 2010.

[30] A. Merabet, K. T. Ahmed, H. Ibrahim, R. Beguenane, and A. M. Y. M. Ghias, "Energy management and control system for laboratory scale microgrid based wind-pv-battery," IEEE Transactions on Sustainable Energy, vol. 8, no. 1, pp. 145-154, Jan 2017.

[31] G. Byeon, T. Yoon, S. M. Oh, and G. Jang, "Energy management strategy of the dc distribution system in buildings using the ev service model," IEEE Transactions on Power Electronics, vol. 28, no. 4, pp. 1544-1554, April 2013

[32] P. Malysz, S. Sirouspour, and A. Emadi, "An optimal energy storage control strategy for grid-connected microgrids," IEEE Transactions on Smart Grid, vol. 5, no. 4, pp. 1785-1796, July 2014.

[33] "IEEE Guide for Optimizing the Performance and Life of Lead-Acid Batteries in Remote Hybrid Power Systems," IEEE Std 1561-2007, pp. C1-25, May 2008. 
[34] M. P. Marietta, M. Graells, and J. M. Guerrero, "A rolling horizon rescheduling strategy for flexible energy in a microgrid," in Energy Conference (ENERGYCON), 2014 IEEE International, May 2014, pp. 1297-1303.

[35] W. Shi, N. Li, C. C. Chu, and R. Gadh, "Real-time energy management in microgrids," IEEE Transactions on Smart Grid, vol. PP, no. 99, pp. $1-1,2015$.

[36] D. E. Olivares, C. A. Caizares, and M. Kazerani, "A centralized energy management system for isolated microgrids," IEEE Transactions on Smart Grid, vol. 5, no. 4, pp. 1864-1875, July 2014.

[37] B. V. Solanki, A. Raghurajan, K. Bhattacharya, and C. A. Caizares, "Including smart loads for optimal demand response in integrated energy management systems for isolated microgrids," IEEE Transactions on Smart Grid, vol. PP, no. 99, pp. 1-10, 2015.

[38] Q. Jiang, M. Xue, and G. Geng, "Energy management of microgrid in grid-connected and stand-alone modes," IEEE Transactions on Power Systems, vol. 28, no. 3, pp. 3380-3389, Aug 2013.

[39] J. Rocabert, A. Luna, F. Blaabjerg, and P. Rodriguez, "Control of power converters in ac microgrids," IEEE Transactions on Power Electronics, vol. 27, no. 11, pp. 4734-4749, Nov 2012.

[40] F. Marra and G. Yang, "Decentralized energy storage in residential feeders with photovoltaics," in Energy Storage for Smart Grids, P. D. Lu, Ed. Boston: Academic Press, 2015, pp. 277 - 294.

[41] G. Giebel and M. Denhard, "Short-term wind power forecasting," in Electric Power Systems: Advanced Forecasting Techniques and Optimal Generation Scheduling, J. P. S. Catalao, Ed. CRC Press, 2012, ch. 5.

[42] D. Linden and T. Reddy, Handbook of batteries. McGraw-Hill handbooks, McGraw-Hill, 2002.

[43] R. Newnham and W. Baldsing, "Advanced management strategies for remote-area power-supply systems," Journal of Power Sources, vol. 133 , no. 1, pp. $141-146,2004$, proceedings of the Tenth Asian Battery Conference.

[44] Intelligent microgrid laboratory. Aalborg University. [Online]. Available: http://www.et.aau.dk/department/laboratory-facilities/ intelligent-microgrid-lab/

[45] A. Ruiz-Alvarez, A. Colet-Subirachs, F. Alvarez-Cuevas Figuerola, O. Gomis-Bellmunt, and A. Sudria-Andreu, "Operation of a utility connected microgrid using an iec 61850-based multi-level management system," IEEE Transactions on Smart Grid, vol. 3, no. 2, pp. 858-865, June 2012.

[46] G. D. Corporation, "General Algebraic Modeling System (GAMS) Release 24.2.1," Washington, DC, USA, 2013. [Online]. Available: url $\{\mathrm{http}: / /$ www.gams.com/ $\}$

[47] T. Kim and W. Qiao, "A hybrid battery model capable of capturing dynamic circuit characteristics and nonlinear capacity effects," IEEE Transactions on Energy Conversion, vol. 26, 2011.

[48] T. Dragicevic, J. Guerrero, J. Vasquez, and D. Skrlec, "Supervisory control of an adaptive-droop regulated $\{\mathrm{DC}\}$ microgrid with battery management capability," IEEE Transactions on Power Electronics, vol. 29, no. 2, pp. 695-706, Feb 2014.

[49] Photovoltaic system. Aalborg University. [Online]. Available: http: //www.et.aau.dk/research-programmes/photovoltaic-systems

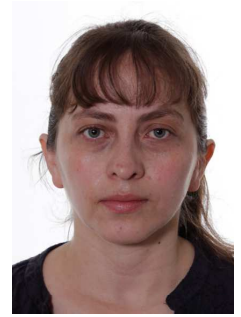

Adriana Luna (S06) received the B.S. degree in electronic engineering in 2006 and M.S. degree in Industrial Automation in 2011, both from Universidad Nacional de Colombia. Since 2010 to 2013 she was Assistant professor in Universidad Libre, Bogotá, Colombia. She was teaching assistant at the Universidad Nacional de Colombia and the Universidad Sergio Arboleda, Bogotá, Colombia in 2011 and 2012 , respectively. She is currently working toward the Ph.D degree in the Microgrid Research Group of the Department of Energy Technology at Aalborg University, Aalborg, Denmark. Her research work focuses on energy management systems of microgrids, and specifically on architectures and algorithms for scheduling and optimization for operation level in microgrids.

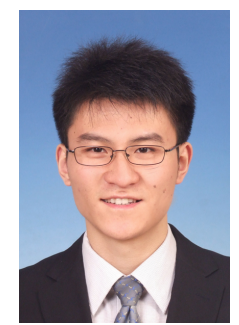

Lexuan Meng (S13, M15) received the B.S. degree in Electrical Engineering and M.S. degree in Electrical Machine and Apparatus from Nanjing University of Aeronautics and Astronautics (NUAA), Nanjing, China, in 2009 and 2012, respectively. In 2015, he received the Ph.D. degree in Power Electronic Systems from Department of Energy Technology, Aalborg University, Denmark. He is currently a postdoctoral researcher in the same department working on flywheel energy storage and onboard electric power systems. His research interests include microgrids, grid integration of energy storage systems, power quality and distributed control. Dr. Meng serves as the Guest Associate Editor of the Special Issue entitled Structured DC Microgrids in the IEEE Journal of Emerging and Selected Topics in Power Electronics. He is a member of IEEE Power Electronics Society (PELS), Industry Applications Society (IAS), Power and Energy Society (PES) and Industrial Electronics Society (IES).

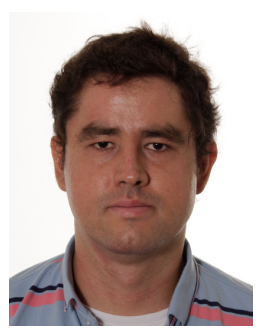

Nelson L. Díaz (S'09-M'17) received the B.S degree in Electronic Engineering from the Universidad Distrital F.J.C in 2008, the M.S. degree in Industrial Automation from the Universidad Nacional de Colombia in 2011, Bogotá, Colombia, and the $\mathrm{Ph} . \mathrm{D}$. degree in Energy Technology from Aalborg University of Denmark in 2017 as part of the Microgrids Research Program. Currently, he is Assistant Professor at the Engineering Faculty of Universidad Distrital F.J.C. of Bogotá Colombia. He is a member of the Research Laboratory of Alternative Energy Sources, Universidad Distrital F.J.C. His current research interests include microgrids and power converters control.

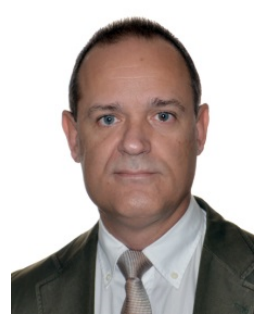

Moisès Graells is Associate Professor of Chemical Engineering at Universitat Politècnica de Catalunya (UPC). He holds a degree in Chemical Science from Universitat Autònoma de Barcelona (UAB, 1989). He was granted by FI/DGU (Generalitat de Catalunya) and obtained his Ph.D. (UPC, 1996). His thesis on Process Systems Engineering was supervised by Emeritus Prof. Luis Puigjaner. Since 1991 he has been a researcher at CEPIMA-UPC and he has participated in several $\mathrm{R}+\mathrm{D}$ national and international projects funded by public (EU programs JOULE, ESPRIT, BRITE, GROWTH, IMS, etc., national programs CICYT, CIRIT, CEDETI, etc.) and private institutions (Hyprotech, Bayer, The CO-LaN). He has also participated in TEMPUS projects for restructuring engineering courses at diverse eastern and former USSR countries (Russia, Ukraine, Bulgaria, etc). He has co-authored more than 80 research papers in international journals; he has co-supervised five Ph.D. theses, published three book chapters in specialized monographs and presented more than 100 communications in national and international meetings. Since 1998 he has been lecturing Chemical Engineering at different UPC schools: Escola Universitria Politècnica de Vilanova i la Geltr (EUPVG), Escola Tcnica Superior dEnginyeria de Industrial de Barcelona (ETSEIB) and Escola Universitària d'Enginyeria Tècnica de Industrial de Barcelona (EUETIB). He is currently at the new Escola dEnginyeria de Barcelona Est (EEBE). He has also been invited lecturer at the Lappeenranta University of Technology (FI), Aalborg University (DK), University of Limmerick (IE), Universidade de Coimbra (PT). His teaching experience includes innovative projects presented to Engineering Education conferences and awarded with the Vicens Vives distinction (Generalitat de Catalunya). He is member of the American Institute of Chemical Engineers (AIChE) and advisor of the UPC AIChE Student Chapter. His research interests have focused on the modeling and optimization of chemical processes, especially batch processes, process integration, waste and energy management, and scheduling and planning. Regarding methodological aspects, his interests are the modeling and optimization techniques: math programming, stochastic and metaheuristic algorithms, machine learning and artificial intelligence, computer aided tools for decision making, and information management. 


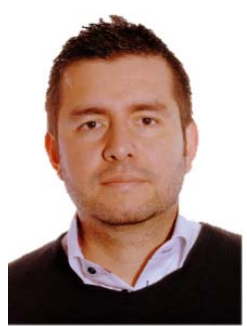

Juan Carlos Vsquez (M12-SM14) received the B.S. degree in electronics engineering from the Autonomous University of Manizales, Manizales, Colombia, and the Ph.D. degree in automatic control, robotics, and computer vision from the Technical University of Catalonia, Barcelona, Spain, in 2004 and 2009, respectively. He was with the Autonomous University of Manizales working as a teaching assistant and the Technical University of Catalonia as a Post-Doctoral Assistant in 2005 and 2008 respectively. In 2011, he was Assistant Professor and from 2014 he is working as an Associate Professor at the Department of Energy Technology, Aalborg University, Denmark where he is the Vice Programme Leader of the Microgrids Research Program (see microgrids.et.aau.dk). From Feb. 2015 to April. 2015 he was a Visiting Scholar at the Center of Power Electronics Systems (CPES) at Virginia Tech and a visiting professor at Ritsumeikan University, Japan. His current research interests include operation, advanced hierarchical and cooperative control, optimization and energy management applied to distributed generation in AC/DC Microgrids, maritime microgrids, advanced metering infrastructures and the integration of Internet of Things and Cyber-Physical Systems into the SmartGrid. He has more than 100 technical papers only in Microgrids in international IEEE conferences and journals.

Dr. Vasquez is currently a member of the IEC System Evaluation Group SEG4 on LVDC Distribution and Safety for use in Developed and Developing Economies, the Renewable Energy Systems Technical Committee TC-RES in IEEE Industrial Electronics, PELS, IAS, and PES Societies.

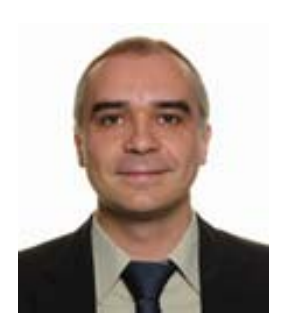

Josep M. Guerrero (S01-M04-SM08-FM15) received the B.S. degree in telecommunications engineering, the M.S. degree in electronics engineering, and the Ph.D. degree in power electronics from the Technical University of Catalonia, Barcelona, in 1997, 2000 and 2003, respectively. Since 2011, he has been a Full Professor with the Department of Energy Technology, Aalborg University, Denmark, where he is responsible for the Microgrid Research Program (www.microgrids.et.aau.dk). From 2012 he is a guest Professor at the Chinese Academy of Science and the Nanjing University of Aeronautics and Astronautics; from 2014 he is chair Professor in Shandong University; from 2015 he is a distinguished guest Professor in Hunan University; and from 2016 he is a visiting professor fellow at Aston University, UK, and a guest Professor at the Nanjing University of Posts and Telecommunications.

His research interests is oriented to different microgrid aspects, including power electronics, distributed energy-storage systems, hierarchical and cooperative control, energy management systems, smart metering and the internet of things for $\mathrm{AC} / \mathrm{DC}$ microgrid clusters and islanded minigrids; recently specially focused on maritime microgrids for electrical ships, vessels, ferries and seaports. Prof. Guerrero is an Associate Editor for the IEEE TRANSACTIONS ON POWER ELECTRONICS, the IEEE TRANSACTIONS ON INDUSTRIAL ELECTRONICS, and the IEEE Industrial Electronics Magazine, and an Editor for the IEEE TRANSACTIONS on SMART GRID and IEEE TRANSACTIONS on ENERGY CONVERSION. He has been Guest Editor of the IEEE TRANSACTIONS ON POWER ELECTRONICS Special Issues: Power Electronics for Wind Energy Conversion and Power Electronics for Microgrids; the IEEE TRANSACTIONS ON INDUSTRIAL ELECTRONICS Special Sections: Uninterruptible Power Supplies systems, Renewable Energy Systems, Distributed Generation and Microgrids, and Industrial Applications and Implementation Issues of the Kalman Filter; the IEEE TRANSACTIONS on SMART GRID Special Issues: Smart DC Distribution Systems and Power Quality in Smart Grids; the IEEE TRANSACTIONS on ENERGY CONVERSION Special Issue on Energy Conversion in Next-generation Electric Ships. He was the chair of the Renewable Energy Systems Technical Committee of the IEEE Industrial Electronics Society. He received the best paper award of the IEEE Transactions on Energy Conversion for the period 2014-2015, and the best paper prize of IEEE-PES in 2015. In 2014 and 2015 he was awarded by Thomson Reuters as Highly Cited Researcher, and in 2015 he was elevated as IEEE Fellow for his contributions on "distributed power systems and microgrids." 